\title{
Black-Hole Spin Dependence in the Light Curves of Tidal Disruption Events
}

\author{
Michael Kesden ${ }^{1, *}$ \\ ${ }^{1}$ Center for Cosmology and Particle Physics, Department of Physics, \\ New York University, New York, New York 10003
}

(Dated: May 2012)

\begin{abstract}
A star orbiting a supermassive black hole can be tidally disrupted if the black hole's gravitational tidal field exceeds the star's self gravity at pericenter. Some of this stellar tidal debris can become gravitationally bound to the black hole, leading to a bright electromagnetic flare with bolometric luminosity proportional to the rate at which material falls back to pericenter. In the Newtonian limit, this flare will have a light curve that scales as $t^{-5 / 3}$ if the tidal debris has a flat distribution in binding energy. We investigate the time dependence of the black-hole mass accretion rate when tidal disruption occurs close enough the black hole that relativistic effects are significant. We find that for orbits with pericenters comparable to the radius of the marginally bound circular orbit, relativistic effects can double the peak accretion rate and halve the time it takes to reach this peak accretion rate. The accretion rate depends on both the magnitude of the black-hole spin and its orientation with respect to the stellar orbit; for orbits with a given pericenter radius in BoyerLindquist coordinates, a maximal black-hole spin anti-aligned with the orbital angular momentum leads to the largest peak accretion rate.
\end{abstract}

\section{INTRODUCTION}

Active galactic nuclei (AGN) are believed to be powered by accretion onto compact objects with masses $M>10^{5} M_{\odot}$ [1]; such objects will inevitably collapse into supermassive black holes (SBHs) on short timescales [2]. Early work [3, 4] conjectured that these AGN were fueled by the tidal disruption of stars passing too close to the SBHs, and that SBHs could grow to their observed masses by accreting debris from such tidal disruption events (TDEs). Although SBHs are now believed to grow primarily by accreting gas driven into galactic centers by tidal torques during galactic mergers [5, 6], interest in TDEs was renewed when it was realized that they could power bright flares lasting several years in otherwise quiescent galaxies 7 .

The Roentgensatellit (ROSAT) observed such a flare in soft X-rays in NGC 5905, a galaxy with no previous indication of nuclear activity [8]. A systematic survey for X-ray flares within the ROSAT All-Sky Survey discovered five such events, implying a rate of $9.1 \times 10^{-6}$ galaxy $^{-1} \mathrm{yr}^{-1}$ consistent with the predicted rate of TDEs 9. Five additional TDE candidates were discovered in the X-ray by the XMM-Newton Slew Survey [10]. TDEs emit in the UV and optical as well; several TDE candidates discovered in the UV by the Galaxy Evolution Explorer (GALEX) were found to have optical counterparts in the Canada-France-Hawaii Telescope Legacy Survey and Panoramic Survey Telescope and Rapid Response System (Pan-STARRS) Medium Deep Survey 11 13. Two new TDE candidates were identified through a series of rigorous cuts on the many optical transients found in Stripe 82 of the Sloan Digital Sky Survey (SDSS); this implies that deeper, higher-cadence surveys such as that

*Electronic address: mhk10@nyu.edu which will be undertaken by the Large Synoptic Survey Telescope (LSST) could find thousands of TDEs per year 14. TDEs can also be observed in hard X-rays if they launch jets pointed towards us; the events Sw J1644+57 15 17 and Sw J2058+05 [18 discovered by the Burst Alert Telescope on the Swift satellite are conjectured to be such relativistic tidal disruption flares.

One of the most exciting applications of observing TDEs is the possibility of measuring SBH spins. The magnitude of a SBH's spin depends sensitively on the manner in which that SBH was assembled. A nonspinning $\mathrm{SBH}$ can attain a dimensionless $\operatorname{spin} a / M \simeq 0.998$ after increasing its mass by a factor of $\sqrt{6}$ through the coherent accretion of gas in the equatorial plane [19, 20]. If a $\mathrm{SBH}$ grows instead through the chaotic accretion of misaligned clumps of gas [21 23], its spin magnitude could be much more modest $(a / M \lesssim 0.2)$. Studies of $\mathrm{SBH}$ spins in the context of hierarchical galaxy formation indicate that a wide range of distributions are possible, depending on one's assumptions about the relative contributions of coherent accretion, chaotic accretion, and binary mergers to SBH spin evolution 24 26]. Simulations in which gas is accreted coherently or chaotically lead to dimensionless spin distributions sharply peaked about unity or zero respectively at all redshifts. Real SBHs probably grow through an as yet undetermined combination of coherent and chaotic accretion, suggesting that almost any distribution of SBH spins is possible. This considerable theoretical uncertainty emphasizes the urgent need for greater observational constraints.

Fortunately, there has recently been tremendous progress measuring SBH spins using X-ray spectroscopy. If an AGN accretion disk is surrounded by a hot, nonthermal corona, a portion of the hard X-rays emitted by this corona may be reprocessed by the colder disk [27, 28]. This hard X-ray irradiation will cause the $6.4 \mathrm{keV}$ iron $\mathrm{K} \alpha$ line in the disk to fluoresce, with much of the flux in this line coming from the innermost regions around 
the SBH [29, 30]. The shape and variability of the line profile is sensitive to general-relativistic effects including SBH spin. Observations of fluorescent iron $\mathrm{K} \alpha$ lines with the XMM-Newton and Suzaku satellites have been used to constrain the spin magnitudes of eight nearby AGN, three of which are near maximal $(a / M \gtrsim 0.98)$ while the remaining five are intermediate $(a / M \simeq 0.7)$ [31, 32.

Despite these impressive new results, this approach to measuring SBH spins is inherently limited to highly accreting AGN whose spins may not reflect the SBH population as a whole. TDEs provide an opportunity to measure the spins of a less biased SBH population, and spin estimates based on TDEs should have different systematic errors than previous approaches. If a TDE launches jets that extract rotational energy from the SBH via the Blandford-Znajek mechanism [33, the observed peak Xray luminosity of the TDE can be used to constrain the SBH spin magnitude [34. If the jet is misaligned with the SBH spin, the Lense-Thirring effect 35 , will cause the jet axis to precess, modulating the observed X-ray emission [36, 37]. Both of these approaches have been applied to the two Swift events J1644+57 and J2058+05. Mean SBH spins may also be estimated from the observed TDE rate 38 40.

In this paper, we focus on yet another way in which TDEs can be used to constrain SBH spins, through the observed light curve of individual events. Several previous authors have investigated relativistic effects during tidal disruption [4144; Laguna et al. 42] provide light curves for TDEs by non-spinning SBHs. If a star with mass $m_{*}$ and radius $R_{*}$ is on an initially parabolic orbit with pericenter $r_{p}$, after tidal disruption roughly half of the stellar mass will become gravitationally bound to the SBH with specific binding energy [7]

$$
E_{\mathrm{tid}}=\frac{G M}{r_{p}^{2}} R_{*}=\beta^{2} \frac{G m_{*}}{R_{*}}\left(\frac{M}{m_{*}}\right)^{1 / 3}
$$

where $\beta \equiv r_{\text {tid }} / r_{p}$ is the penetration factor, $r_{\text {tid }}=$ $\left(M / m_{*}\right)^{1 / 3} R_{*}$ is the tidal radius, and the binding energy is defined as the positive energy required to unbind the system. Newtonian orbits with specific binding energy $E$ have orbital period

$$
t=2 \pi G M(2 E)^{-3 / 2},
$$

implying that the debris will return to pericenter a time

$$
\begin{aligned}
t_{\mathrm{tid}} & =\beta^{-3} \frac{\pi}{m_{*}}\left(\frac{M R_{*}^{3}}{2 G}\right)^{1 / 2} \\
& =0.11 \mathrm{yr} \beta^{-3}\left(\frac{M}{10^{6} M_{\odot}}\right)^{1 / 2}\left(\frac{m_{*}}{M_{\odot}}\right)^{-1}\left(\frac{R_{*}}{R_{\odot}}\right)^{3 / 2}(3)
\end{aligned}
$$

after tidal disruption [45]. Internal shocks during this pericenter passage will cause the debris to settle into an axisymmetric torus on this timescale. If the viscosity is high enough to allow accretion on a similar or shorter timescale, Eq. (2) implies that the TDE luminosity $L \propto$ $|d E / d t| \propto t^{-5 / 3}$ provided the energy distribution $d m / d E$ of the tidal debris is constant 7, 46. The light curves of several TDE candidates have been reasonably well fit by $t^{-5 / 3}$ power laws in the optical, UV, and X-ray [12, 13, 15, 47.

The $L \propto t^{-5 / 3}$ dependence of TDE light curves rests on two assumptions: the constancy of $d m / d E$ and the validity of the Newtonian relation (2) between the specific binding energy and orbital period. The first of these assumptions was investigated by Lodato, King, and Pringle [48. (hereafter LKP09), who found that $d m / d E$ did indeed depend on the adiabatic index $\gamma$ of the tidally disrupted star. This dependence on $\gamma$ is reflected in the earliest portions of the TDE light curve; Gezari et al. 13. showed that the TDE candidate PS1-10jh discovered by Pan-STARRS and GALEX is more consistent with a fully convective star or degenerate core with $\gamma=5 / 3$ than a solar-type star with $\gamma=4 / 3$.

The second assumption was investigated by Haas et al. 49, who performed six numerical-relativity simulations of the tidal disruption of a white dwarf by a $10^{3} M_{\odot}$ intermediate-mass black hole (IMBH). The IMBH was nonspinning in the first simulation, and in the remaining five simulations had a spin with the same magnitude $(a / M=0.6)$ but different orientations with respect to the orbital angular momentum and initial position of the white dwarf. They found that the prompt accretion rate at $t \lesssim 6$ s after tidal disruption could vary by almost two orders of magnitude as a function of spin direction. Numerical-relativity simulations are computationally expensive however, and it is not feasible to perform such simulations for the long timescales $t_{\text {tid }}$ given by Eq. (3) over which debris continues to fall back to pericenter. Haas et al. [49 instead estimate the fallback time for each fluid element at the end of their simulations assuming that subsequent motion is on Keplerian ballistic trajectories [50. They find, similar to LKP09, that $d m / d t \propto t^{-5 / 3}$ at sufficiently late times.

In this paper, we use the assumption that tidal debris moves on Kerr geodesics to calculate mass accretion rates as a function of time throughout the full fallback regime. Our focus will be on the most massive SBHs where the tidal radius $r_{\text {tid }}$ is comparable to the Schwarzschild radius $r_{s}$; the extreme mass ratios $\left(m_{*} / M \lesssim 10^{-6}\right)$ imply that full numerical relativity is not required leading to a vast reduction in computational expense. In Sec. III. we review the Newtonian calculation of the mass accretion rate given by LKP09. We then propose a relativistic generalization of this calculation in Sec. III] Using this new relativistic framework, we present the accretion rate as a function of SBH mass, spin, and initial stellar orbit in Sec. IV] We discuss the implications of our results and future applications of this framework in Sec. V] For the reader's convenience, we have summarized the Kerr metric and the use of Fermi normal coordinates in Appendices $\mathrm{A}$ and $\mathrm{B}$. 


\section{NEWTONIAN ACCRETION RATE}

A simplified model of stellar tidal disruption in the Newtonian limit is presented in Sec. 2 of LKP09. This model is premised on the existence of a clear hierarchy between the specific orbital kinetic energy $E_{\text {orb }}$ of the initial star, the energy $E_{\text {tid }}$ gained or lost during tidal disruption, and the self-binding energy $E_{\text {bin }}$ of the star [50. These energy scales are given by

$$
\begin{aligned}
& E_{\mathrm{orb}}=\frac{G M}{r_{p}}, \\
& E_{\mathrm{tid}}=\frac{G M}{r_{p}^{2}} R_{*}=\beta q^{1 / 3} E_{\mathrm{orb}}, \\
& E_{\mathrm{bin}}=\frac{G m_{*}}{R_{*}}=\beta^{-1} q^{2 / 3} E_{\mathrm{orb}},
\end{aligned}
$$

where $r_{p}$ is the pericenter and $q \equiv m_{*} / M \ll 1$ is the mass ratio. This hierarchy implies that to zeroth order in $q$, the specific binding energy of a fluid element of the tidal debris is the same as that of the center of mass of the initial star. At $\mathcal{O}\left(q^{1 / 3}\right)$, a fluid element located at $\mathbf{r}$ receives a correction to its specific energy

$$
\Delta E=\frac{G M}{r_{p}^{2}} \hat{\mathbf{r}}_{p} \cdot\left(\mathbf{r}-\mathbf{r}_{p}\right)
$$

where $\mathbf{r}_{p}$ points from the black hole to the center of the star at pericenter, and vectors with hats above them are unit vectors. This correction is of order $E_{\text {tid }}$. The kinetic energy of the fluid element in the star's center-of-mass frame is of order $E_{\mathrm{bin}}$, as is the gravitational binding energy of the fluid element to the star. These can therefore be safely neglected to this order in $q$.

Given this hierarchy of energy scales, Rees [7] approximated the distribution in specific energy of the tidal debris of a star on an initially parabolic orbit $(E=0)$ as

$$
\begin{aligned}
\frac{d m}{d E} & =\frac{m_{*}}{2 E_{\mathrm{tid}}} & & |E|<E_{\mathrm{tid}} \\
& =0 & & |E|>E_{\mathrm{tid}} .
\end{aligned}
$$

As described in Sec. I1 this approximation leads to a mass accretion rate that begins a time $t_{\text {tid }}$ after tidal disruption given by Eq. (3) and falls of as $t^{-5 / 3}$ thereafter.

LKP09 move beyond this level of approximation, explicitly calculating the energy distribution of the tidal debris to $\mathcal{O}\left(q^{1 / 3}\right)$ for different polytropic equations of state $P=C \rho^{\gamma}$. They begin by solving the Lane-Emden equation to determine the density profile of the initial star [51]

$$
\frac{1}{\xi^{2}} \frac{d}{d \xi}\left(\xi^{2} \frac{d \Theta}{d \xi}\right)=-\Theta^{n}
$$

where $n \equiv(\gamma-1)^{-1}$ is the polytropic index and $\Theta$ and $\xi$ are dimensionless variables which give the star's density

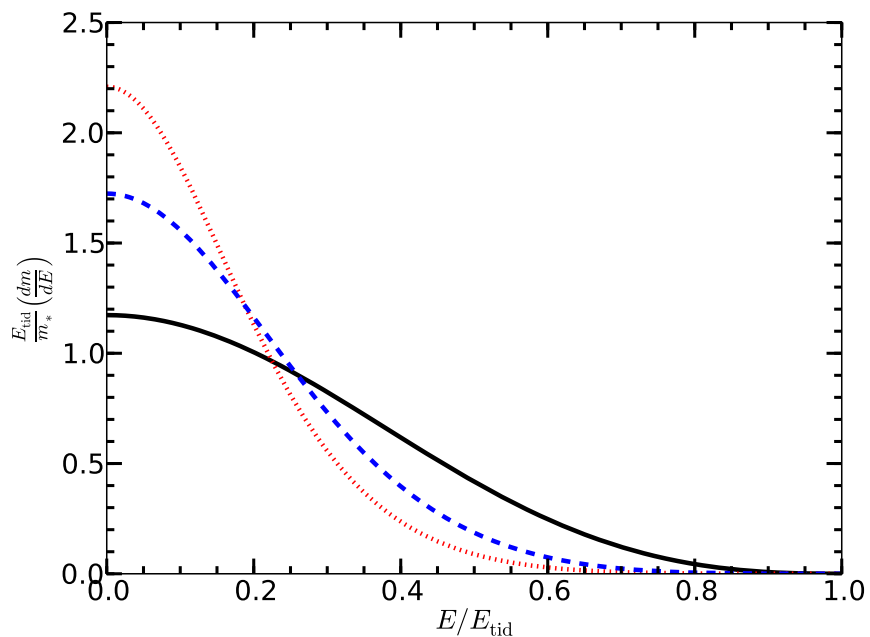

FIG. 1: The distribution $d m / d E$ of the specific binding energy $E$ for the tidal debris of a star on an initially parabolic orbit. The solid black curve corresponds to a adiabatic index $\gamma=$ $5 / 3$ appropriate for a fully convective star, while the dashed blue and dotted red curves correspond to $\gamma=1.4$ and $4 / 3$ respectively.

profile through the relations

$$
\begin{aligned}
\rho & =\rho_{c} \Theta^{n}, \\
r & =b \xi, \\
b & =\left[\frac{(n+1) C \rho_{c}^{\gamma-2}}{4 \pi G}\right]^{1 / 2},
\end{aligned}
$$

where $\rho_{c}$ is the star's central density. The Lane-Emden equation (7) can be solved numerically by beginning at the star's center $\xi=0$ where $\Theta(0)=1, d \Theta / d \xi(0)=0$, then integrating to the star's surface at $\xi_{1}$ defined by the condition $\Theta\left(\xi_{1}\right)=0$. If the specific binding energy of a fluid element is related to its position by Eq. (5), the energy distribution $d m / d E$ of the tidal debris is given in terms of the star's initial density profile $\Theta(\xi)$ by

$$
\frac{E_{\mathrm{tid}}}{m_{*}} \frac{d m}{d E}=\frac{\int_{E / E_{\mathrm{tid}}}^{1} \Theta^{n} x d x}{2 \int_{0}^{1} \Theta^{n} x^{2} d x},
$$

where $x \equiv \xi / \xi_{1}$ is a rescaled radial coordinate. This dimensionless energy distribution for several adiabatic indices $\gamma$ is shown in Fig. 1 1 which reproduces the left panel of Fig. 2 of LKP09.

If the orbital period $t$ of a fluid element is given in terms of its specific binding energy by Eq. (2), the rate $d m / d t$ at which mass falls back to pericenter and is subsequently accreted is given by

$$
\frac{t_{0}}{m_{*}} \frac{d m}{d t}=\frac{1}{3} \frac{r_{p}}{R_{*}}\left(\frac{E_{\mathrm{tid}}}{m_{*}} \frac{d m}{d E}\right)\left(\frac{t}{t_{0}}\right)^{-5 / 3},
$$

where $t_{0} \equiv 2 \pi\left(r_{p}^{3} / G M\right)^{1 / 2}$ is the period of an orbit with semi-major axis $r_{p}$. We plot this dimensionless accretion 


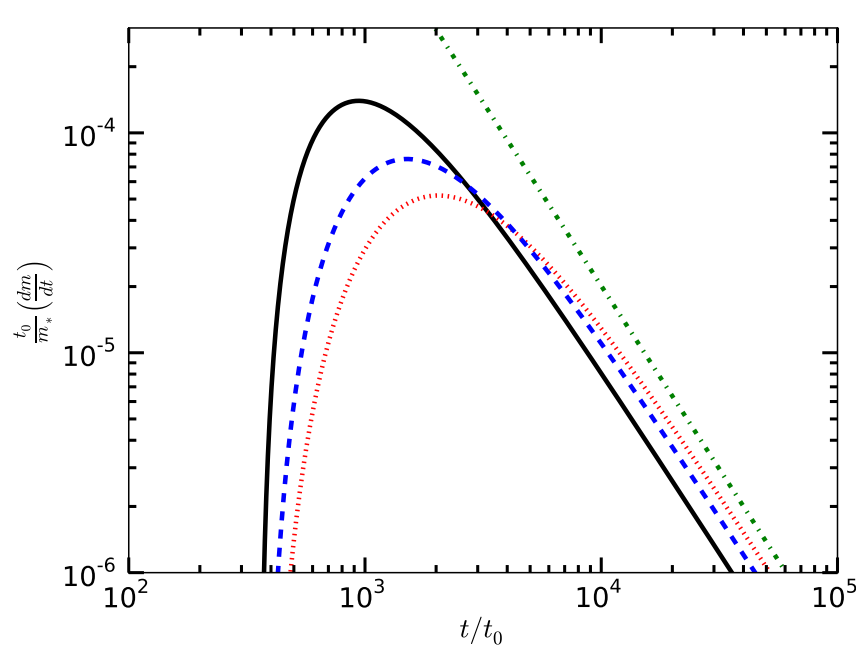

FIG. 2: The rate $d m / d t$ at which the debris of a tidally disrupted star falls back to pericenter and is subsequently accreted. The time $t$ is given in units of $t_{0} \equiv 2 \pi\left(r_{p}^{3} / G M\right)^{1 / 2}$, the period of an orbit with semi-major axis $r_{p}$. As in Fig. 1 the solid black, dashed blue, and dotted red curves correspond to adiabatic indices $\gamma=5 / 3,1.4$, and $4 / 3$ respectively. The dot-dashed green line shows the canonical $t^{-5 / 3}$ dependence predicted by [7, 46].

rate for $r_{p}=100 R_{*}$ and several values of $\gamma$ in Fig. 2, a reproduction of the middle panel of Fig. 2 of LKP09.

After introducing this simplified model of tidal disruption in Sec. 2 of their paper, LKP09 devote the rest of the paper to testing its validity using a series of non-relativistic smoothed-particle-hydrodynamics (SPH) simulations. They find that Eq. (9) accurately describes the energy distribution of the tidal debris after the initial density profile of the star has been homologously expanded by a factor of 1.6 to 2.5 depending on the adiabatic index $\gamma$. This expansion reflects the stretching of the star by tidal forces prior to its arrival at pericenter. The mass accretion rate $d m / d t$ measured in the simulations also confirms the predictions shown in Fig. 2. a stiffer equation of state (larger $\gamma$ ) leads to a higher peak accretion rate and a faster approach to the latetime $t^{-5 / 3}$ behavior. The success of the LKP09 model provides a strong motivation for developing a relativistic version of this model appropriate to TDEs where the pericenter distance $r_{p}$ is comparable to the Schwarzschild radius $r_{S}=2 G M / c^{2}$ of the $\mathrm{SBH}$.

\section{RELATIVISTIC ACCRETION RATE}

Kerr geodesics differ from Newtonian orbits in several important respects that need to be incorporated into our new relativistic model for tidal disruption. We provide a brief review of Kerr geodesics in Appendix A One difference is that Kerr geodesics generally do not close, unlike bound orbits about a Newtonian point mass. Since we are interested in the rate at which tidal debris returns to pericenter, we will focus on the radial period and ignore that the second pericenter will generally occur at different values of $\theta$ and $\phi$ than the pericenter at which the star was initially disrupted. This precession may affect the timescale on which the tidal debris settles into an accretion disk, but this process is beyond the scope of the current work.

A second difference between Kerr geodesics and Newtonian orbits is that the radial period depends not only on the specific energy $E$, as in Eq. (2), but also on the specific angular momentum $L_{z}$ and Carter constant $Q$ as can be seen from Eq. A10b. Determining the accretion rate $d m / d t$ will therefore require not just the energy distribution $d m / d E$, but the full joint distribution $d^{3} \mathrm{~m} / d E d L_{z} d Q$ for all three constants of motion. Also, as we do not have a nice relation like Eq. (2) for the radial period, we will have to integrate Eqs. A10 directly for each fluid element of the tidal debris.

The greatest difference between our relativistic model and the LKP09 model is the manner in which we determine the distribution $d^{3} \mathrm{~m} / d E d L_{z} d Q$. In the Newtonian limit, the specific energy

$$
E=c^{2}+\frac{1}{2} v^{2}+\Phi(r)
$$

separates into three distinct components: the rest-mass energy $c^{2}$, the kinetic energy $v^{2} / 2$, and the gravitationalpotential energy $\Phi(r)$. The rest-mass energy is neglected in the LKP09 model because it does not affect the orbital motion in the Newtonian limit. Although the kinetic energy of the center of mass is equal in magnitude to the gravitational-potential energy for a parabolic orbit, the relative velocity $v$ between fluid elements at pericenter can be neglected because it leads to energy corrections of order $E_{\text {bin }}$, which according to Eq. (4) are suppressed by a factor $q^{1 / 3} / \beta^{2}$ compared to the dominant corrections of order $E_{\text {tid }}$ arising from the gravitational potential. The constants of motion $E, L_{z}$, and $Q$ given by Eqs. A5 and (A8) do not neatly separate into components that depend only on $r$ or $v$ as in Eq. (11), however the recognition that we can set the relative velocity $v=0$ provides the key for calculating the relativistic distribution $d^{3} \mathrm{~m} / d E d L_{z} d Q$.

It is often convenient to use Fermi normal coordinates when calculating quantities near a point (such as the center of mass of a tidally disrupting star) that moves along a Kerr geodesic. We review the use of these coordinates in Appendix B. If a fluid element is located at a fixed position $X^{i}$ in Fermi normal coordinates, its relative velocity with respect to the origin (the center of mass) will vanish [52]:

$$
v^{\alpha}=\lambda_{0}^{\beta} \nabla_{\beta}\left(X^{i} \lambda_{i}^{\alpha}\right)=X^{i} \lambda_{0}^{\beta} \nabla_{\beta} \lambda_{i}^{\alpha}=0 .
$$

The final equality follows because our basis vectors $\lambda_{A}^{\alpha}$ were constructed to be parallel transported along the central geodesic $\left(\lambda_{0}^{\beta} \nabla_{\beta} \lambda_{i}^{\alpha}=0\right)$. The difference in specific 
energy between this fluid element and one located at the origin is given by

$$
\begin{aligned}
\Delta E & =X^{i} \lambda_{i}^{\alpha} \nabla_{\alpha} E \\
& =X^{i} \lambda_{i}^{\alpha} \nabla_{\alpha}\left[-g_{\beta \gamma} \lambda_{0}^{\beta}\left(\frac{\partial}{\partial t}\right)^{\gamma}\right] \\
& =-g_{\beta \gamma} \lambda_{0}^{\beta} X^{i} \lambda_{i}^{\alpha} \nabla_{\alpha}\left(\frac{\partial}{\partial t}\right)^{\gamma} \\
& =-g_{\beta \gamma} \lambda_{0}^{\beta} X^{i} \lambda_{i}^{\alpha} \Gamma_{\alpha t}^{\gamma} .
\end{aligned}
$$

The second equality follows from the definition of $E$ in Eq. A5a. The third equality follows from the compatibility of the metric with the derivative operator, the commutation of coordinate vector fields $\left(\lambda_{i}^{\alpha} \nabla_{\alpha} \lambda_{0}^{\beta}=\right.$ $\lambda_{0}^{\alpha} \nabla_{\alpha} \lambda_{i}^{\beta}$ ) [52], and the parallel transport of the basis vectors. The final equality follows from the definition of the Christoffel symbols. Similar calculations show that

$$
\begin{aligned}
\Delta L_{z} & =X^{i} \lambda_{i}^{\alpha} \nabla_{\alpha} L_{z} \\
& =g_{\beta \gamma} \lambda_{0}^{\beta} X^{i} \lambda_{i}^{\alpha} \Gamma_{\alpha \phi}^{\gamma},
\end{aligned}
$$

and

$$
\begin{aligned}
\Delta K & =X^{i} \lambda_{i}^{\alpha} \nabla_{\alpha} K \\
& =2 X^{i}\left[\lambda_{0}^{\alpha} \lambda_{0}^{\beta} \lambda_{i}^{\gamma} \nabla_{\gamma}\left(\Sigma l_{\alpha} n_{\beta}\right)-r \lambda_{i}^{r}\right],
\end{aligned}
$$

where $K$ is defined in Eq. A8 and $l^{\alpha}$ and $n^{\alpha}$ are given by Eq. A7). We can then compute the difference

$$
\Delta Q=\Delta K-2\left(L_{z}-a E\right)\left(\Delta L_{z}-a \Delta E\right)
$$

in the Carter constant $Q$ of the fluid element compared to the center of mass.

Our prescription for calculating the mass fallback rate in the relativistic limit is as follows:

(1) Choose an orbit for the tidally disrupting star characterized by its 4 -velocity $\lambda_{0}^{\alpha}$ at pericenter and constants of motion $E, L_{z}$, and $Q$.

(2) Determine the density profile $\rho(r)$ of the star from its mass $m_{*}$, radius $R_{*}$, and adiabatic index $\gamma$ by solving the Lane-Emden equation (7).

(3) Use $\rho(r)$ to determine a distribution of positions $X^{i}$ for the fluid elements in Fermi normal coordinates.

(4) Use Eqs. 13), 14, 15, and (B3) to determine the positions $x^{\alpha}$ of the fluid elements in BoyerLindquist coordinates and their constants of motion $E+\Delta E, L_{z}+\Delta L_{z}$, and $Q+\Delta Q$.

(5) Determine from their positions and constants of motion whether each fluid element is on a geodesic that plunges directly into the event horizon, escapes to infinity, or becomes bound to the SBH.

(6) If a fluid element is on a bound orbit, integrate the equations of motion A10 out to apocenter beginning from $t=0$; the final time $t_{f}$ will be half of the radial period.
(7) Create a histogram of the resulting distribution of orbital periods to determine the fallback accretion rate $d m / d t$, similar to that depicted in Fig. 2 in the Newtonian limit.

\section{RESULTS}

We begin by presenting our predictions for the distribution $d^{3} m / d E d L_{z} d Q$ in Sec. IV A this is intermediate step (4) in the prescription given above. While this provides some insight into how $\mathrm{SBH}$ spin affects the tidal debris, those interested only in the corresponding fallback accretion rates $d m / d t$ can skip ahead to Sec. IV B.

\section{A. Distribution of orbital constants}

Eqs. (13) - 16 reveal that $\Delta E, \Delta L_{z}$, and $\Delta Q$ for each fluid element are inner products of the position $X^{i}$ of that fluid element in Fermi normal coordinates with covariant derivatives in the direction of the spatial basis vectors $\lambda_{i}^{\alpha}$. In the Newtonian limit, these quantities are inner products of $\mathbf{r}-\mathbf{r}_{p}$ with flat-space gradients. We have already seen this in Eq. (5), the Newtonian limit of Eq. 13). This implies that the projected distributions $d m / d E, d m / d L_{z}$, and $d m / d Q$ will have the exact same shape as the Newtonian distribution shown in Fig. 1. The width of the relativistic distribution $d m / d E$ is defined as

$$
\sigma_{E} \equiv\left|\lambda_{i}^{\alpha} \nabla_{\alpha} E\right| R_{*} ;
$$

the widths $\sigma_{L_{z}}$, and $\sigma_{Q}$ are found by replacing $E$ with $L_{z}$ or $Q$ in Eq. (17). These widths depend on the SBH mass $M$, dimensionless spin $a / M$, and the orbit of the tidally disrupted star. We explore this dependence in this section.

In the Newtonian limit, $\sigma_{E} \rightarrow E_{\text {tid }}$ given by Eq. (1). We did not calculate the corresponding widths $L_{z \text {,tid }}$ and $Q_{\text {tid }}$ of $d m / d L_{z}$ and $d m / d Q$ in Sec. II, as these quantities did not enter into the Newtonian orbital period given by Eq. (2). As the radial period for bound orbits in the Kerr spacetime does depend on $L_{z}$ and $Q$, we now calculate these widths. In the Newtonian limit,

$$
\begin{aligned}
\Delta \mathbf{L}_{N} & =\left(\mathbf{r}-\mathbf{r}_{p}\right) \times \mathbf{v} \\
& =\left(\frac{2 G M}{r_{p}}\right)^{1 / 2}\left\{\left[\left(\mathbf{r}-\mathbf{r}_{p}\right) \cdot \hat{\mathbf{r}}_{p}\right] \hat{\mathbf{L}}_{N}-\left[\left(\mathbf{r}-\mathbf{r}_{p}\right) \cdot \hat{\mathbf{L}}_{N}\right] \hat{\mathbf{r}}_{p}\right\}
\end{aligned}
$$

implying that

$$
\begin{aligned}
\Delta L_{z} & =\Delta \mathbf{L}_{N} \cdot \hat{\mathbf{z}} \\
& =\left(\frac{2 G M}{r_{p}}\right)^{1 / 2}\left(\hat{\mathbf{r}}_{p} \cos \iota-\hat{\mathbf{L}}_{N} \cos \theta_{p}\right) \cdot\left(\mathbf{r}-\mathbf{r}_{p}\right)
\end{aligned}
$$

and

$$
\begin{aligned}
\Delta Q & =2 \Delta \mathbf{L}_{N} \cdot\left[\left(\mathbf{L}_{N} \cdot \hat{\mathbf{x}}\right) \hat{\mathbf{x}}+\left(\mathbf{L}_{N} \cdot \hat{\mathbf{y}}\right) \hat{\mathbf{y}}\right] \\
& =4 G M\left(\hat{\mathbf{r}}_{p} \sin ^{2} \iota+\hat{\mathbf{L}}_{N} \cos \theta_{p} \cos \iota\right) \cdot\left(\mathbf{r}-\mathbf{r}_{p}\right) .
\end{aligned}
$$




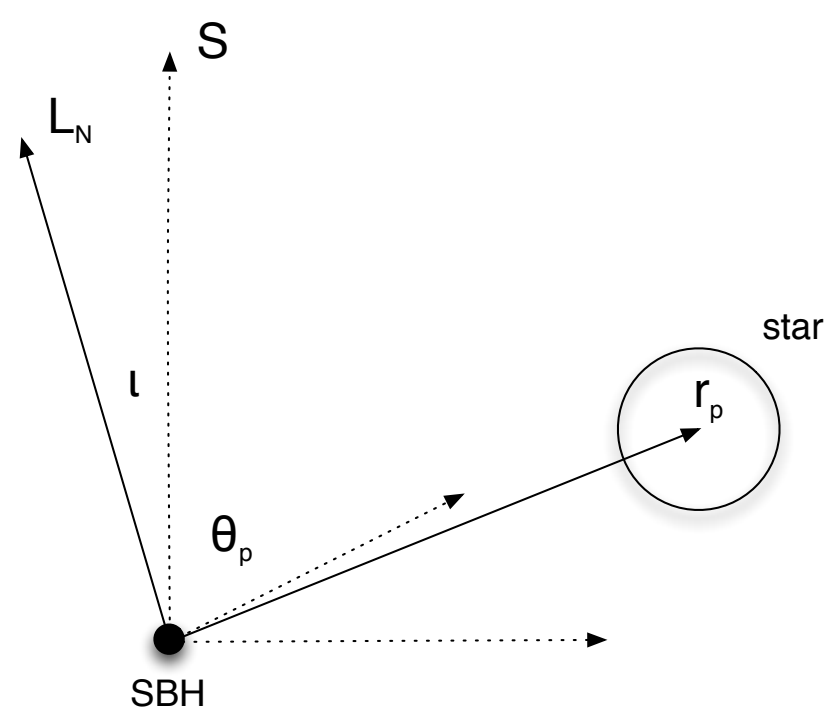

FIG. 3: The $\mathrm{SBH}$ is located at the origin with its spin $\mathbf{S}$ pointing along the $\mathrm{z}$ axis. The inclination $\iota$ is the angle between $\mathbf{S}$ and the star's orbital angular momentum $\mathbf{L}_{N}$, and $\theta_{p}$ is the angle between $\mathbf{S}$ and the position $\mathbf{r}_{p}$ of the star at pericenter.

where $\mathbf{L}_{N}$ is the orbital angular momentum and the angles $\theta_{p}$ and $\iota$ are defined in Fig. 3 . Eqs. (19) and (20) allow us to define the widths of the $L_{z}$ and $Q$ distributions in the Newtonian limit:

$$
\begin{aligned}
L_{z, \text { tid }} & =\left(\frac{2 G M}{r_{p}}\right)^{1 / 2}\left(\cos ^{2} \iota+\cos ^{2} \theta_{p}\right)^{1 / 2} R_{*}, \\
Q_{\mathrm{tid}} & =4 G M\left(\sin ^{4} \iota+\cos ^{2} \theta_{p} \cos ^{2} \iota\right)^{1 / 2} R_{*} .
\end{aligned}
$$

Examining the ratio $\sigma_{X} / X_{\text {tid }}$ of the widths of the distribution $d m / d X$ of a quantity $X$ in the relativistic and Newtonian limits will allow us to assess the accuracy of the Newtonian calculation.

\section{Equatorial orbits}

Equatorial orbits $\left(\theta_{p}=90^{\circ}\right)$ can be either prograde $\left(\iota=0^{\circ}\right)$ or retrograde $\left(\iota=180^{\circ}\right)$. These orbits have $Q=0$, and Eq. (21b) shows that the tidal debris will also have $Q=0$ in the Newtonian limit. This holds for the relativistic calculation as well. If we define

$$
f_{r} \equiv 1-\frac{2 G M}{r_{p}}
$$

then for nonspinning SBHs,

$$
\begin{aligned}
\frac{\sigma_{E}}{E_{\mathrm{tid}}} & =f_{r}^{-1 / 2}, \\
\frac{\sigma_{L_{z}}}{L_{z, \mathrm{tid}}} & =\left[\frac{\cos ^{2} \iota+f_{r}^{-1} \cos ^{2} \theta_{p}}{\cos ^{2} \iota+\cos ^{2} \theta_{p}}\right]^{1 / 2} .
\end{aligned}
$$

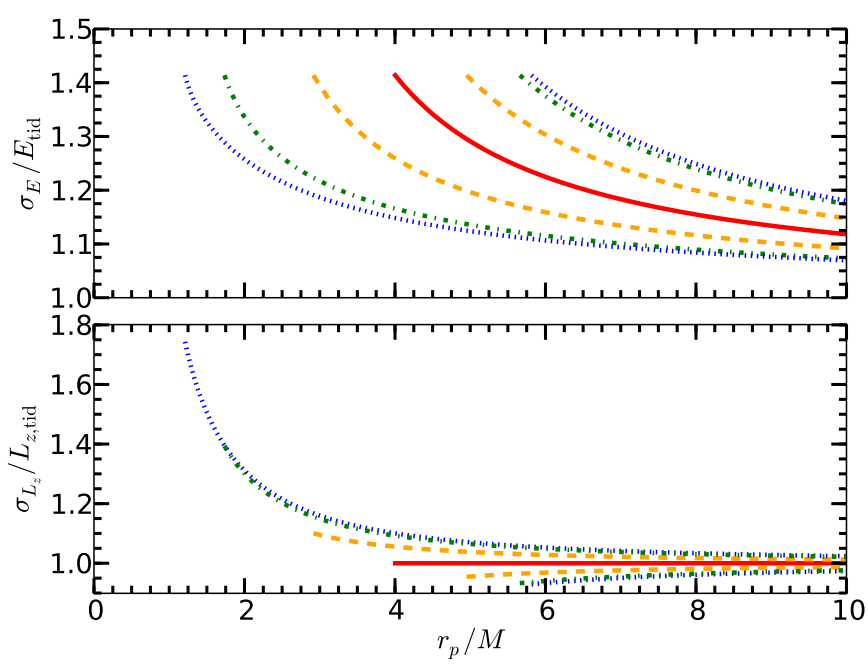

FIG. 4: The widths $\sigma_{E}$ and $\sigma_{L_{z}}$ of the projected distributions $d m / d E$ and $d m / d L_{z}$ as functions of the pericenter radius $r_{p} / M$. These are normalized by the widths $E_{\text {tid }}$ and $L_{z, \text { tid }}$ of the corresponding Newtonian distributions. The solid red curves are for nonspinning black holes, while the dashed orange, dot-dashed green, and dotted blue curves are for dimensionless spins $a / M=0.5,0.9$, and 0.99 respectively. For each value of the spin, the curve extending to smaller values of $r_{p}$ corresponds to prograde $\left(\iota=0^{\circ}\right)$ orbits while the other curve corresponds to retrograde $\left(\iota=180^{\circ}\right)$ orbits.

We show these ratios for several additional values of the dimensionless spin $a / M$ in Fig. 4. At fixed values of $r_{p}, \sigma_{E}$ decreases (increases) with $a / M$ on prograde (retrograde) orbits. However, at the radius $r_{\mathrm{mb}}$ of the marginally bound circular orbit (minimum value of $r_{p}$ for each curve), $\sigma_{E} / E_{\mathrm{tid}}=\sqrt{2}$ independent of SBH spin. By contrast, the ratio $\sigma_{L_{z}} / L_{z \text {, tid }}$ at $r_{\mathrm{mb}}$ depends sharply on $a / M$. Although $\sigma_{L_{z}} / L_{z \text {,tid }}=1$ all the way down to $r_{\mathrm{mb}}=4 M$ for nonspinning SBHs, it increases to 2 (decreases to $\sim 0.929)$ as $a / M \rightarrow 1$ for prograde (retrograde) orbits.

\section{Inclined orbits}

Inclined orbits have $0^{\circ}<\iota<180^{\circ}$; for orbits with $L_{z}<0$ it is convenient to define a supplementary inclination

$$
\cos \iota^{\prime} \equiv \frac{-L_{z}}{\sqrt{Q+L_{z}^{2}}}
$$

such that $\iota^{\prime}=180^{\circ}-\iota$. If we define a complementary angle $\theta_{p}^{\prime} \equiv 90^{\circ}-\theta_{p}$, Eq. A10c implies that for parabolic $(E=1)$ orbits

$$
\begin{aligned}
-\iota \leq \theta_{p}^{\prime} \leq+\iota & \iota \leq 90^{\circ} \\
-\iota^{\prime} \leq \theta_{p}^{\prime} \leq+\iota^{\prime} & \iota>90^{\circ}
\end{aligned}
$$




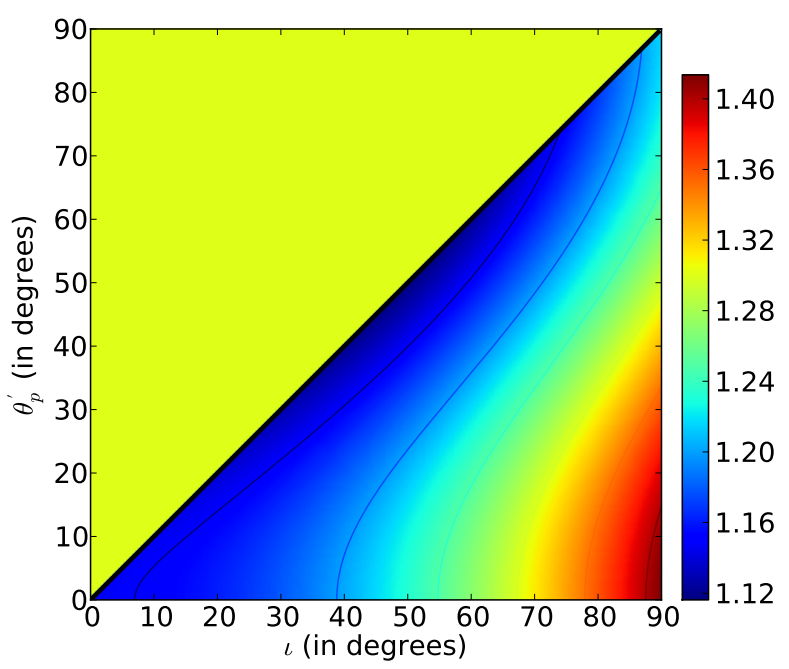

FIG. 5: The ratio $\sigma_{E} / E_{\text {tid }}$ for orbits with $r_{p}=4 M, a / M=$ 0.99 , and $\iota \leq 90^{\circ}$ as a function of inclination $\iota$ and latitude $\theta_{p}^{\prime}=90^{\circ}-\bar{\theta}_{p}$. The yellow region above the diagonal is unphysical as it corresponds to a portion of parameter space forbidden by the inequality $25 \mathrm{a}$.

The relativistic corrections $\sigma_{X} / X_{\text {tid }}$ to the width of a distribution $d m / d X$ will depend on both $\iota$ and $\theta_{p}$ for inclined orbits, in addition to $r_{p} / M$ and $a / M$.

We show $\sigma_{E} / E_{\text {tid }}$ for inclined orbits with $r_{p}=4 M$, $a / M=0.99$, and $\iota \leq 90^{\circ}$ in Fig. 5. The origin of this figure corresponds to the same orbit as that shown by the point at $r_{p}=4 M$ on the lower dotted blue curve in the upper panel of Fig. 4. We see that the relativistic correction $\sigma_{E} / E_{\text {tid }}$ is largest when tidal disruption occurs in the equatorial plane $\left(\theta_{p}^{\prime}=0^{\circ}\right)$ and increases with inclination $\iota$. Although not apparent from this figure, for higher spins and smaller radii the ratio $\sigma_{E} / E_{\text {tid }}$ can exceed 1.5 on highly inclined orbits, greater than the value $\sim \sqrt{2}$ found on the marginally bound orbits in Fig. 4

The ratio $\sigma_{E} / E_{\text {tid }}$ for orbits with $r_{p}=6 M, a / M=$ 0.99 , and $\iota>90^{\circ}$ is shown in Fig. 6. We must use a larger value of $r_{p}$ than in Fig. 5 , as the marginally bound orbits with $L_{z}<0$ have larger radii. The origin of this figure corresponds to the same orbit as that shown by the point at $r_{p}=6 \mathrm{M}$ on the upper dotted blue curve in the upper panel of Fig. 4. As was the case for $\iota<90^{\circ}$, the relativistic correction is greatest in the equatorial plane and increases with inclination $\iota$. Figs. 5 and 6 are both consistent with our impression from Fig. 4 that the value of $\sigma_{E} / E_{\text {tid }}$ largely depends on the difference between $r_{p}$ and the radius of the marginally bound orbit for that spin and inclination.

While $E_{\text {tid }}$ is independent of orbital inclination, both the numerator and denominator of the ratio $\sigma_{L_{z}} / L_{z \text {,tid }}$ depend on $\iota$ and $\theta_{p}$. Eq. 21a) and 23b show that although both $\sigma_{L_{z}}$ and $L_{z, \text { tid }} \rightarrow 0$ as $\iota \rightarrow 90^{\circ}, \theta_{p} \rightarrow 90^{\circ}$ for $a / M=0$, their ratio is undefined in this limit. For $a / M>0$, the location at which $\sigma_{L_{z}}=0$ shifts to

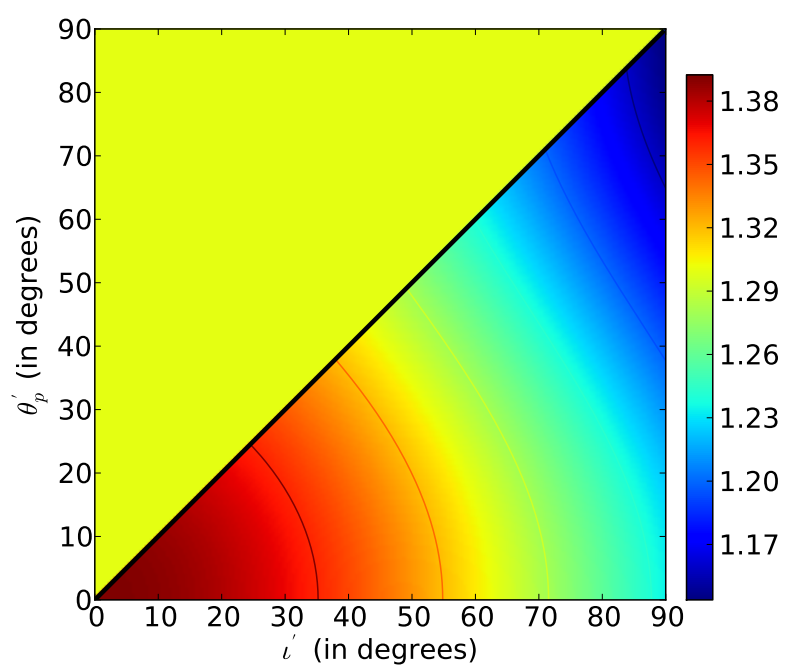

FIG. 6: The ratio $\sigma_{E} / E_{\text {tid }}$ for orbits with $r_{p}=6 M, a / M=$ 0.99 , and $\iota>90^{\circ}$ as a function of supplementary inclination $\iota^{\prime}=180^{\circ}-\iota$ and latitude $\theta_{p}^{\prime}$. The portion of the plot above the diagonal is unphysical.



FIG. 7: The ratio $\sigma_{L_{z}} / L_{z \text {,tid }}$ for orbits with $r_{p}=4 M, a / M=$ 0.99 , and $\iota \leq 85^{\circ}$ as a function of inclination $\iota$ and latitude $\theta_{p}^{\prime}$. The region of the plot above the diagonal is unphysical.

$\iota_{0}^{\prime}(a / M)<90^{\circ}, \theta_{p}=90^{\circ}$ while the Newtonian denominator $L_{z, \text { tid }}$ of Eq. 21a remains unchanged. This implies that the ratio $\sigma_{L_{z}} / L_{z \text {,tid }}$ will have a pole at $\iota=90^{\circ}$, $\theta_{p}^{\prime}=0^{\circ}$, as can be seen in Fig. 7 where we show this ratio for orbits with $r_{p}=4 M, a / M=0.99$, and $\iota \leq 85^{\circ}$. The figure is cut off above $\iota=85^{\circ}$ to avoid this pole. The presence of this pole is also apparent in the contour plot of $\sigma_{L_{z}} / L_{z \text {,tid }}$ for orbits with $r_{p}=6 M, a / M=0.99$, and $\iota^{\prime} \leq 85^{\circ}$ shown in Fig. 8. We also see that $\sigma_{L_{z}} \rightarrow 0$ as one approaches $\iota_{0}^{\prime} \simeq 80^{\circ}, \theta_{p}^{\prime}=0^{\circ}$.

Finally we come to the width of distribution of the Carter constant $Q$, which for nonspinning SBHs is given 


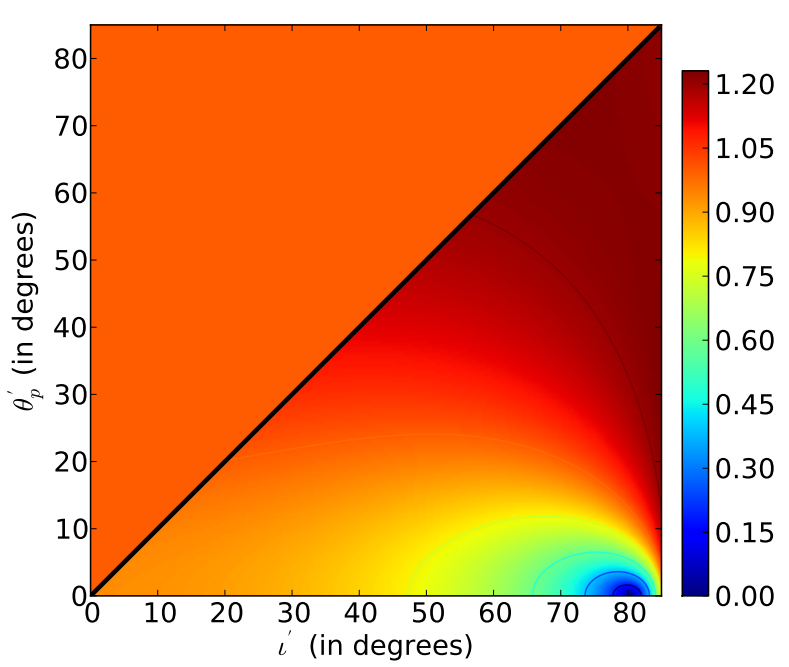

FIG. 8: The ratio $\sigma_{L_{z}} / L_{z \text {,tid }}$ for orbits with $r_{p}=6 M, a / M=$ 0.99 , and $\iota \geq 95^{\circ}$ as a function of supplemental inclination $\iota^{\prime}=180^{\circ}-\iota$ and latitude $\theta_{p}^{\prime}$. The region of the plot above the diagonal is unphysical.

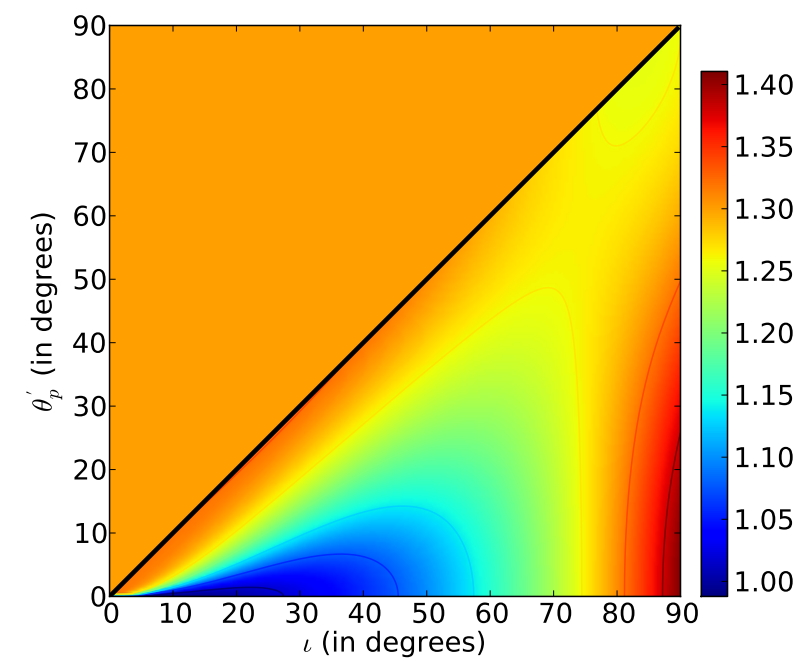

FIG. 9: The ratio $\sigma_{Q} / Q_{\text {tid }}$ for orbits with $r_{p}=4 M, a / M=$ 0.99 , and $\iota \leq 90^{\circ}$ as a function of inclination $\iota$ and latitude $\theta_{p}^{\prime}$. The region of the plot above the diagonal is unphysical.

by

$$
\frac{\sigma_{Q}}{Q_{\mathrm{tid}}}=\left[f_{r}^{-1} \frac{\sin ^{4} \iota+f_{r}^{-1} \cos ^{2} \theta_{p} \cos ^{2} \iota}{\sin ^{4} \iota+\cos ^{2} \theta_{p} \cos ^{2} \iota}\right]^{1 / 2} .
$$

We show this ratio for orbits with $r_{p}=4 M, a / M=0.99$, and $\iota \leq 90^{\circ}$ in Fig. 9. This contour plot differs qualitatively from the nonspinning result 26 in that $\sigma_{Q} / Q_{\text {tid }}$ is maximized at $\iota=90^{\circ}, \theta_{p}^{\prime}=0^{\circ}$ instead of near $\iota=0^{\circ}$, $\theta_{p}^{\prime}=0^{\circ}$. We show $\sigma_{Q} / Q_{\text {tid }}$ for orbits with $r_{p}=6 M$, $a / M=0.99$, and $\iota \geq 90^{\circ}$ in Fig. 10 . This figure more closely resembles the nonspinning result 26 , although

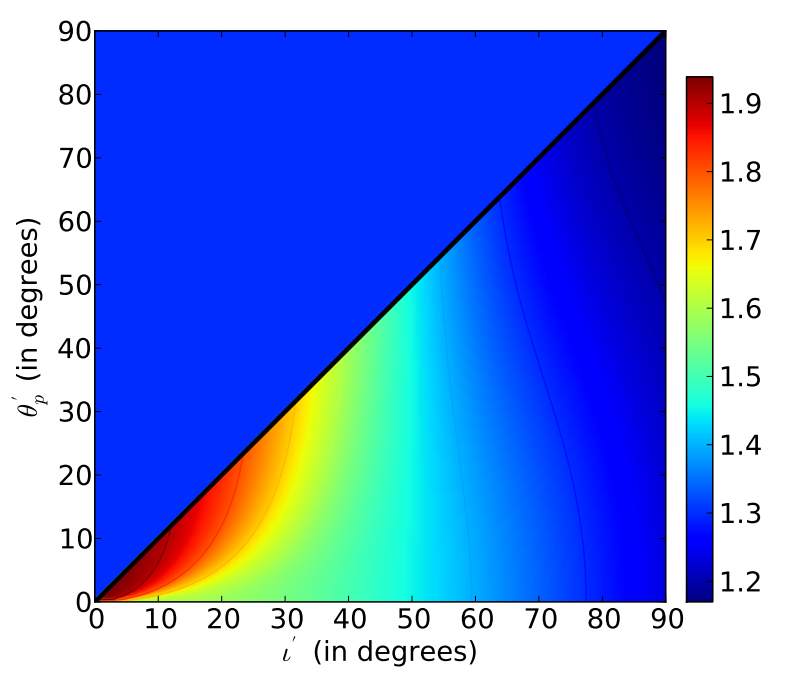

FIG. 10: The ratio $\sigma_{Q} / Q_{\text {tid }}$ for orbits with $r_{p}=6 M, a / M=$ 0.99 , and $\iota \geq 90^{\circ}$ as a function of supplemental inclination $\iota^{\prime}=180^{\circ}-\iota$ and latitude $\theta_{p}^{\prime}$. The region of the plot above the diagonal is unphysical.

$\sigma_{Q}^{2}$ no longer separates into two distinct terms, only one of which is dependent on $\theta_{p}$.

\section{B. Fallback accretion rate}

We now present the fallback accretion rate $d m / d t$ for stellar tidal disruptions with different values of the $\mathrm{SBH}$ mass $M, \mathrm{SBH}$ spin $a / M$, pericenter distance $r_{p}$, orbital inclination $\iota$, and polar angle $\theta_{p}$ at pericenter. We fix $m_{*}=M_{\odot}, R_{*}=R_{\odot}$, and $\gamma=5 / 3$ throughout this section, as our focus is on relativistic effects that are independent of these parameters. We measure time in units of $t_{\mathrm{tid}}$ given by Eq. (3), and $r_{p}$ in units of $M$ (where $G=c=1)$.

\section{Equatorial orbits}

In Fig. 11, we show the fallback accretion rate $d m / d t$ for TDEs of stars on equatorial $\left(\theta_{p}=90^{\circ}\right)$, parabolic $(E=0)$ orbits with $r_{p}=6 M$ by SBHs with $M=10^{8} M_{\odot}$. The different curves correspond to different values of the $\mathrm{SBH}$ spin and either prograde or retrograde orbits as described in the caption. We see that relativistic corrections can substantially affect the accretion rate for stars with such small pericenters. The peak accretion rate $d m_{\text {peak }} / d t$ can be almost twice as large as the Newtonian prediction, and can occur at a time $t_{\text {peak }}$ only half that of the Newtonian prediction. SBH spin magnitude and direction also strongly affect the accretion rate; large prograde $\left(\iota=0^{\circ}\right)$ spins reduce $d m_{\text {peak }} / d t$ and delay $t_{\text {peak }}$, while large retrograde spins $\left(\iota=180^{\circ}\right)$ have the opposite effect. The jaggedness in the curves results from the fi- 


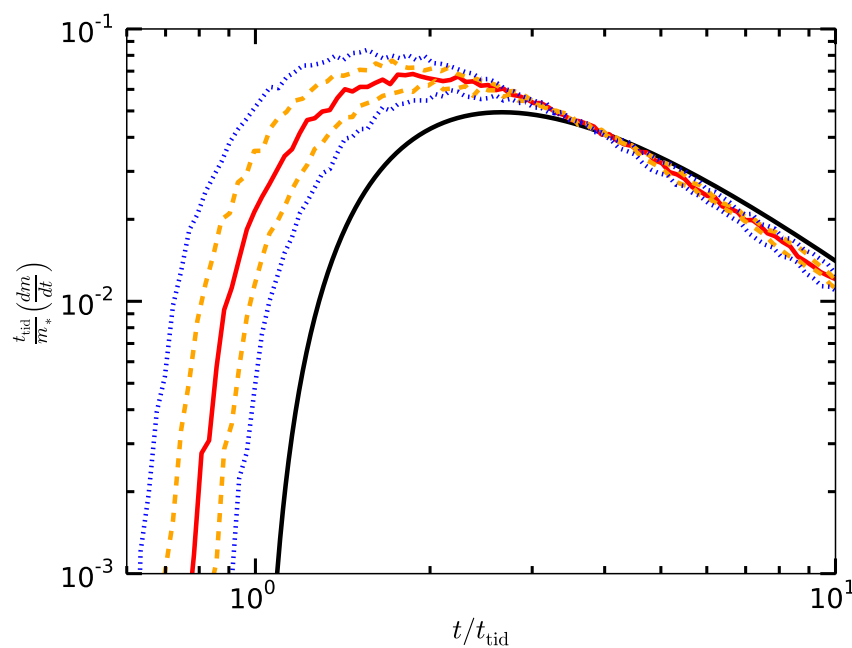

FIG. 11: The dimensionless fallback accretion rate $\left(t_{\mathrm{tid}} / m_{*}\right) d m / d t$ as a function of time $t / t_{\mathrm{tid}}$ following the tidal disruption of a stars on equatorial orbits with $r_{p}=6 M$ by SBHs with $M=10^{8} M_{\odot}$. The smooth, solid black curve is the Newtonian prediction shown in Fig. 2. The solid red curve is the relativistic prediction for a non-spinning $\mathrm{SBH}$. The dashed orange (dotted blue) curves correspond to spin magnitudes $a / M=0.5(0.99)$ respectively. The curves of each type with lower (higher) peak accretion rates correspond to orbital inclinations $\iota=0^{\circ}\left(180^{\circ}\right)$.

nite number of particles $\left(N=10^{6}\right)$ and finite proper time step $\left(\Delta \tau=10^{3} M\right)$. Each curve took approximately 15 minutes to prepare on a single-processor $2.2 \mathrm{GHz}$ laptop; better optimization and additional computational resources could allow a more thorough exploration of parameter space with greater accuracy.

In Fig. 12, we again show the fallback accretion rate for equatorial orbits with $r_{p}=6 M$, but have reduced the SBH mass from $10^{8} M_{\odot}$ to $10^{6} M_{\odot}$. By measuring time in units of $t_{\mathrm{tid}}$, we have eliminated the the dependence of the Newtonian prediction on $M$ and $r_{p}$. The magnitude of the relativistic effects is similar to that in Fig. 11 for the prograde orbits, but the accretion rate has been sharply cut off at early times for the retrograde orbits. This cutoff occurs because the mostly tightly bound particles (those which would have the shortest orbital periods) have lost so much energy $E$ and angular momentum $L_{z}$ that they are directly swallowed by the SBH event horizon. This effect is very small for $a / M=0.5$, where only $0.021 \%$ of the star's initial mass is directly swallowed (a slight reduction in the retrograde dashed orange curve at the earliest times is just barely noticeable). The cutoff is much more pronounced for $a / M=0.9$ and 0.99 shown by the dot-dashed green and dotted blue curves respectively. In these cases $21.4 \%$ and $34.2 \%$ of the initial stellar mass is directly captured. This directly captured material will have little opportunity to radiate, and should therefore not contribute to observed TDE light curves.

In our dimensionless units, the Eddington accretion

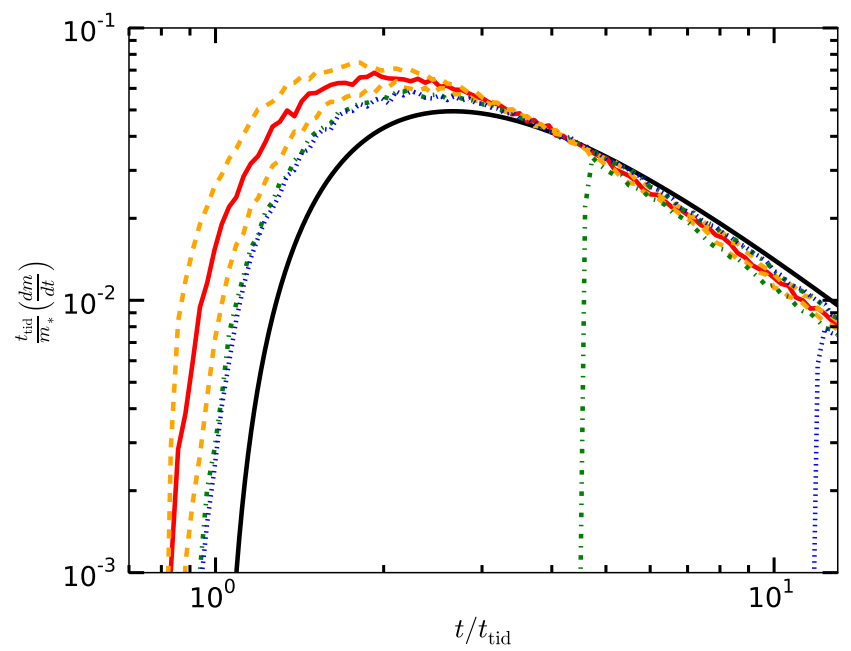

FIG. 12: The dimensionless fallback accretion rate $\left(t_{\text {tid }} / m_{*}\right) d m / d t$ as a function of time $t / t_{\text {tid }}$ following the tidal disruption of a stars on equatorial orbits with $r_{p}=6 M$ by SBHs with $M=10^{6} M_{\odot}$. The smooth, solid black curve is the Newtonian prediction. The solid red curve is the relativistic prediction for a non-spinning SBH. The dashed orange, dot-dashed green, and dotted blue curves correspond to spin magnitudes $a / M$ of $0.5,0.9$, and 0.99 respectively. The curves of each type with higher (lower) accretion rates at late times correspond to orbital inclinations $\iota=0^{\circ}\left(180^{\circ}\right)$.

rate is

$$
\begin{aligned}
\frac{t_{\mathrm{tid}}}{m_{*}} \frac{d m_{\mathrm{Edd}}}{d t}= & \frac{4 \pi^{2} m_{p}}{\eta m_{*}^{2} \sigma_{T} c} \beta^{-3}\left(\frac{G M^{3} R_{*}^{3}}{2}\right)^{1 / 2} \\
= & 51.4\left(\frac{\eta}{0.1}\right)^{-1}\left(\frac{m_{*}}{M_{\odot}}\right)^{-1}\left(\frac{R_{*}}{R_{\odot}}\right)^{-3 / 2} \\
& \times\left(\frac{r_{p}}{6 M}\right)^{3}\left(\frac{M}{10^{8} M_{\odot}}\right)^{7 / 2}
\end{aligned}
$$

where $m_{p}$ is the proton mass, $\eta$ is the radiative efficiency, and $\sigma_{T}$ is the Thomson cross section. While the peak accretion rates for $10^{8} M_{\odot}$ SBHs as shown in Fig. 11 are comfortably sub-Eddington, those for $10^{6} M_{\odot} \mathrm{SB} \overline{\mathrm{Hs}}$ as shown in Fig. 12 are highly super-Eddington. It is therefore unlikely that the observed TDE luminosity for $M=10^{6} M_{\odot}$ will track the fallback accretion rate at early times which are most sensitive to SBH spin. More massive SBHs have peak accretion rates below the Eddington limit, but are only capable of tidally disrupting stars when

$$
\frac{r_{p}}{M} \lesssim \frac{r_{\mathrm{tid}}}{M} \simeq 47.1\left(\frac{M}{10^{6} M_{\odot}}\right)^{-2 / 3}\left(\frac{m_{*}}{M_{\odot}}\right)^{-1 / 3}\left(\frac{R_{*}}{R_{\odot}}\right) .
$$

SBHs as massive as $10^{8} M_{\odot}$ will directly capture many stars without tidally disrupting them, although relativistic effects allow TDEs for $M \lesssim 10^{9} M_{\odot}$ despite this Newtonian estimate 38 40]. These considerations suggest 


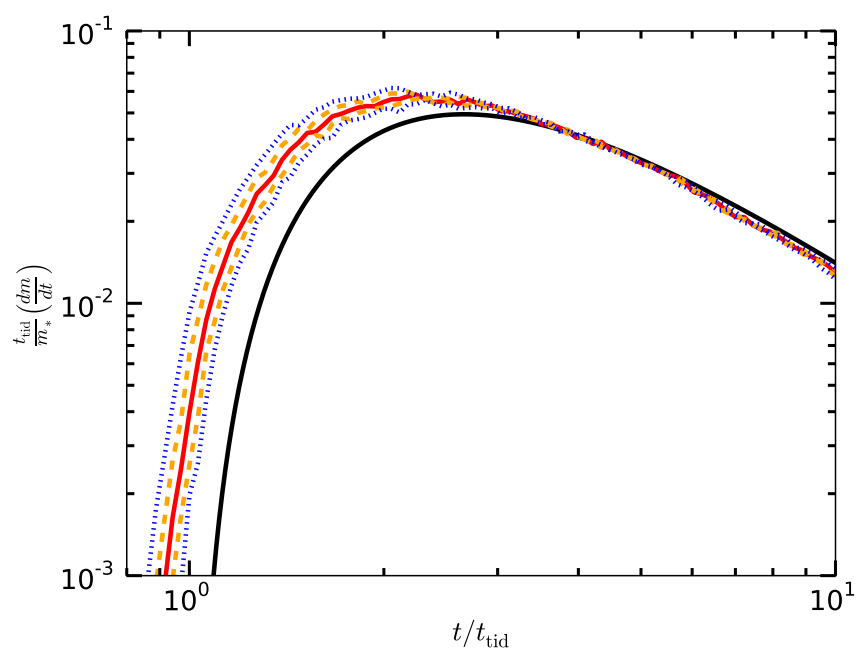

FIG. 13: The dimensionless fallback accretion rate $\left(t_{\mathrm{tid}} / m_{*}\right) d m / d t$ as a function of time $t / t_{\mathrm{tid}}$ following the tidal disruption of a stars on equatorial orbits with $r_{p}=12 M$ by SBHs with $M=10^{7} M_{\odot}$. The smooth, solid black curve is the Newtonian prediction. The solid red curve is the relativistic prediction for a non-spinning SBH. The dashed orange (dotted blue) curves correspond to spin magnitudes $a / M$ of 0.5 (0.99). The curves of each type with lower (higher) peak accretion rates correspond to orbital inclinations $\iota=0^{\circ}\left(180^{\circ}\right)$.

that $M \simeq 10^{7} M_{\odot}$ may be a sweet spot for attempts to constrain SBH spins with TDE light curves, which is fortuitous since SBHs of this mass are fairly ubiquitous at the modest redshifts where TDEs are readily observable.

We show the fallback accretion rates for TDEs by a SBH with $M=10^{7} M_{\odot}$ in Fig. 13 . The pericenter for the TDEs shown in this figure has been increased to $r_{p}=12 M$ to show how relativistic effects fall off with distance. Comparing this figure to Fig. 11, we see that relativistic corrections increase $d m_{\text {peak }} / d t$ and reduce $t_{\text {peak }}$ by at most $\sim 25 \%$, and SBH spin induces similar corrections to these quantities at the $\sim 25 \%$ level.

\section{Inclined orbits}

Tidally disrupted stars are scattered onto their initial orbits at large radii where the influence of the $\mathrm{SBH}$ spin is negligible. One should therefore expect a flat distribution in $\cos \iota$ in the range $-1 \leq \cos \iota \leq 1$. We now consider how the fallback accretion rate depends on $\iota$ and the polar angle $\theta_{p}$ at pericenter for inclined orbits. We show $d m / d t$ for fixed spin magnitude $a / M=0.99$ and $\theta_{p}=90^{\circ}$ but different orbital inclinations $\iota$ in Fig. 14. The Newtonian prediction in this figure is identical to that in Fig. 11, and the dotted blue (dot-dashed cyan) curves corresponding to $\iota=0^{\circ}\left(180^{\circ}\right)$ are identical to the dotted blue curves in Fig. 11. The remaining curves correspond to inclined

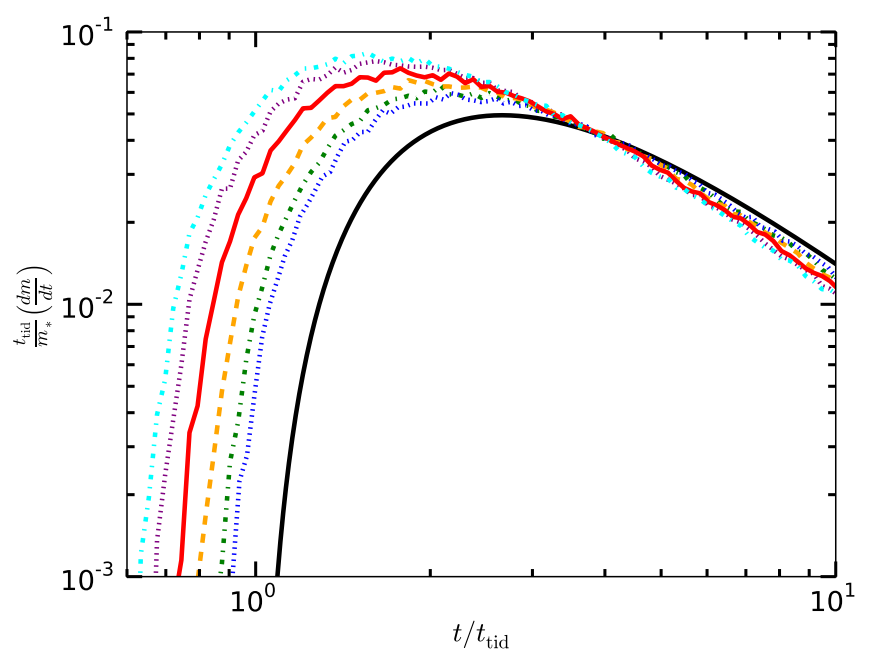

FIG. 14: The dimensionless fallback accretion rate $\left(t_{\mathrm{tid}} / m_{*}\right) d m / d t$ as a function of time $t / t_{\mathrm{tid}}$ following the tidal disruption of a stars on inclined orbits with $r_{p}=6 M$ by SBHs with $M=10^{8} M_{\odot}$ and $a / M=0.99$. The smooth, solid black curve is the Newtonian prediction. The remaining curves correspond to relativistic predictions with polar angle $\theta_{p}=90^{\circ}$ but different values of the orbital inclination $\iota$. The peak accretion rate $d m_{\text {peak }} / d t$ increases with $\iota$; with increasing values of $d m_{\text {peak }} / d t$, the dotted blue, dot-dashed green, dashed orange, solid red, dotted purple, and dot-dashed cyan curves correspond to $\iota=0^{\circ}, 45^{\circ}, 75^{\circ}, 105^{\circ}, 135^{\circ}$, and $180^{\circ}$.

orbits that were discussed in detail in Sec. IVA2, In terms of the relativistic correction $\sigma_{E} / E_{\mathrm{tid}}$ to the width of the energy distribution, increasing $\iota$ from $0^{\circ}$ to $180^{\circ}$ while keeping $\theta_{p}=90^{\circ}$ fixed corresponds to moving from left to right along the $\mathrm{x}$-axis of Fig. 5 , then moving from right to left along the x-axis of Fig. 6. The ratio $\sigma_{E} / E_{\text {tid }}$ increases with $\iota$ along this path, qualitatively implying that tidal debris falls deeper into the SBH's potential well with shorter radial periods $t / M$. Although in the relativistic limit the radial period depends on $L_{z}$ and $Q$ in addition to $E$, consideration of $E$ alone seems to illustrate the qualitative dependence on $\iota$.

We show the dependence of the fallback accretion rate on the polar angle at pericenter $\theta_{p}$ in Fig. 15. As in the previous figure, Fig. 15 depicts TDEs of stars with pericenter $r_{p}=6 M$ by a SBH with $M=10^{8} M_{\odot}$ and $a / M=0.99$. The orbital inclination is fixed at $\iota=80^{\circ}$, but the latitude $\theta_{p}^{\prime}=90^{\circ}-\theta_{p}$ is varied from $0^{\circ}$ to $60^{\circ}$. In terms of the ratio $\sigma_{E} / E_{\mathrm{tid}}$, this corresponds to moving upwards along the vertical line $\iota=80^{\circ}$ in Fig. 5. The ratio $\sigma_{E} / E_{\text {tid }}$ decreases as we move along this path, implying that the tidal debris becomes less tightly bound to the SBH with longer radial periods $t / M$. This qualitatively accounts for the trend seen in Fig. 15 , that $d m / d t$ is shifted to later times as $\theta_{p}$ decreases ( $\theta_{p}^{\prime}$ increases). Although not shown, this same trend holds for $\iota>90^{\circ}$ as one would expect from consideration of Fig. 6. Our results for the peak accretion rate $d m_{\text {peak }} / d t$ and the 


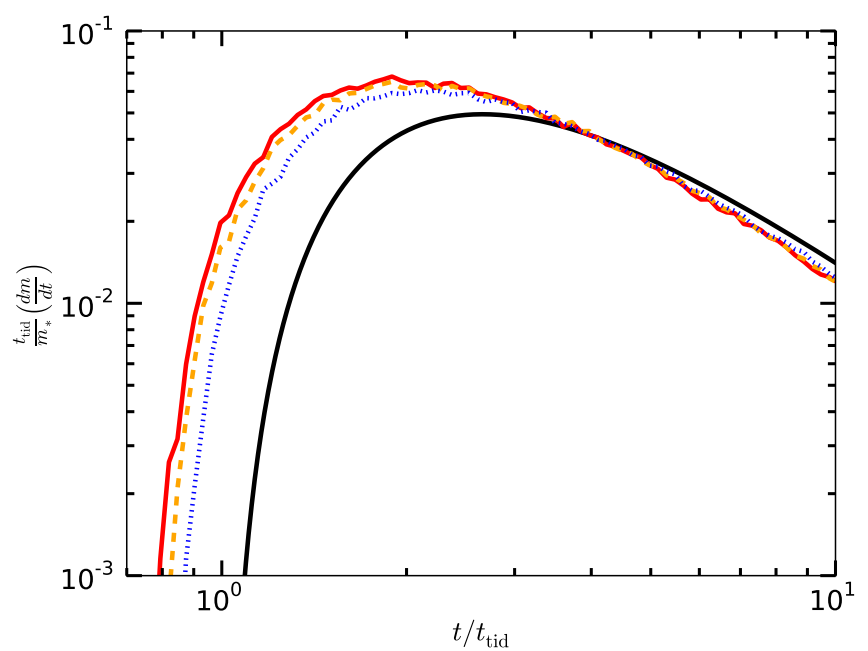

FIG. 15: The dimensionless fallback accretion rate $\left(t_{\mathrm{tid}} / m_{*}\right) d m / d t$ as a function of time $t / t_{\mathrm{tid}}$ following the tidal disruption of a stars on inclined orbits with $r_{p}=6 M$ by SBHs with $M=10^{8} M_{\odot}$ and $a / M=0.99$. The smooth, solid black curve is the Newtonian prediction. The remaining curves correspond to relativistic predictions with inclination $\iota=80^{\circ}$ but different values of the polar angle at pericenter $\theta_{p}$. The peak accretion rate $d m_{\text {peak }} / d t$ increases with $\theta_{p}$; with increasing values of $d m_{\text {peak }} / d t$, the dotted blue, dashed orange, and solid red, curves correspond to $\theta_{p}=30^{\circ}, 60^{\circ}$, and $90^{\circ}$.

time $t_{\text {peak }}$ at which this peak occurs are summarized in Table [.

\section{DISCUSSION}

Our primary goals in this paper have been to establish a theoretical framework for investigating stellar tidal disruption deep in the relativistic regime and to conduct a preliminary survey of the many dimensional parameter space associated with this problem. Our starting point for this framework was the Newtonian model proposed in LKP09, in which a star's orbit and density profile were used to determine the distribution of orbital energy of the tidal debris and hence the fallback accretion rate. The gravitational potential of a Newtonian point mass is spherically symmetric, implying that for a given stellar profile the only physically significant parameters are the SBH mass $M$ and the pericenter $r_{p}$. The Kerr spacetime however is only axisymmetric, increasing the number of required orbital parameters to include the $\mathrm{SBH}$ spin $a / M$, the orbital inclination $\iota$, and the polar angle at pericenter $\theta_{p}$. The radial period of the tidal debris now depends on the angular momentum $L_{z}$ and Carter constant $Q$ in addition to the energy $E$. Fermi normal coordinates allow us to determine the distribution of these orbital constants for a given stellar profile and orbit, and

\begin{tabular}{ccccccc}
\hline \hline$M$ & $a / M$ & $r_{p}$ & $\iota$ & $\theta_{p}$ & $\frac{t_{\text {peak }}}{t_{\text {tid }}}$ & $\frac{t_{\text {tid }}}{m_{*}} \frac{d m_{\text {peak }}}{d t}$ \\
\hline \hline $10^{8} M_{\odot}$ & 0.0 & $6 M$ & $0^{\circ}$ & $90^{\circ}$ & 1.87 & 0.0683 \\
$10^{8} M_{\odot}$ & 0.5 & $6 M$ & $0^{\circ}$ & $90^{\circ}$ & 2.04 & 0.0645 \\
$10^{8} M_{\odot}$ & 0.5 & $6 M$ & $180^{\circ}$ & $90^{\circ}$ & 1.72 & 0.0766 \\
$10^{8} M_{\odot}$ & 0.99 & $6 M$ & $0^{\circ}$ & $90^{\circ}$ & 2.17 & 0.0596 \\
$10^{8} M_{\odot}$ & 0.99 & $6 M$ & $180^{\circ}$ & $90^{\circ}$ & 1.56 & 0.0838 \\
$10^{6} M_{\odot}$ & 0.0 & $6 M$ & $0^{\circ}$ & $90^{\circ}$ & 1.92 & 0.0685 \\
$10^{6} M_{\odot}$ & 0.5 & $6 M$ & $0^{\circ}$ & $90^{\circ}$ & 2.11 & 0.0638 \\
$10^{6} M_{\odot}$ & 0.5 & $6 M$ & $180^{\circ}$ & $90^{\circ}$ & 1.81 & 0.0747 \\
$10^{6} M_{\odot}$ & 0.9 & $6 M$ & $0^{\circ}$ & $90^{\circ}$ & 2.24 & 0.0595 \\
$10^{6} M_{\odot}$ & 0.9 & $6 M$ & $180^{\circ}$ & $90^{\circ}$ & 4.73 & 0.0335 \\
$10^{6} M_{\odot}$ & 0.99 & $6 M$ & $0^{\circ}$ & $90^{\circ}$ & 2.24 & 0.0585 \\
$10^{6} M_{\odot}$ & 0.99 & $6 M$ & $180^{\circ}$ & $90^{\circ}$ & 12.33 & 0.0079 \\
$10^{7} M_{\odot}$ & 0.0 & $12 M$ & $0^{\circ}$ & $90^{\circ}$ & 2.25 & 0.0583 \\
$10^{7} M_{\odot}$ & 0.5 & $12 M$ & $0^{\circ}$ & $90^{\circ}$ & 2.28 & 0.0564 \\
$10^{7} M_{\odot}$ & 0.5 & $12 M$ & $180^{\circ}$ & $90^{\circ}$ & 2.17 & 0.0599 \\
$10^{7} M_{\odot}$ & 0.99 & $12 M$ & $0^{\circ}$ & $90^{\circ}$ & 2.31 & 0.0558 \\
$10^{7} M_{\odot}$ & 0.99 & $12 M$ & $180^{\circ}$ & $90^{\circ}$ & 2.05 & 0.0617 \\
$10^{8} M_{\odot}$ & 0.99 & $6 M$ & $45^{\circ}$ & $90^{\circ}$ & 2.09 & 0.0636 \\
$10^{8} M_{\odot}$ & 0.99 & $6 M$ & $75^{\circ}$ & $90^{\circ}$ & 1.89 & 0.0665 \\
$10^{8} M_{\odot}$ & 0.99 & $6 M$ & $105^{\circ}$ & $90^{\circ}$ & 1.78 & 0.0735 \\
$10^{8} M_{\odot}$ & 0.99 & $6 M$ & $135^{\circ}$ & $90^{\circ}$ & 1.61 & 0.0781 \\
$10^{8} M_{\odot}$ & 0.99 & $6 M$ & $80^{\circ}$ & $30^{\circ}$ & 2.15 & 0.0606 \\
$10^{8} M_{\odot}$ & 0.99 & $6 M$ & $80^{\circ}$ & $60^{\circ}$ & 2.02 & 0.0651 \\
$10^{8} M_{\odot}$ & 0.99 & $6 M$ & $80^{\circ}$ & $90^{\circ}$ & 1.89 & 0.0680 \\
\hline \hline & & & & & &
\end{tabular}

TABLE I: Peak accretion rate $d m_{\text {peak }} / d t$ and the time $t_{\text {peak }}$ at which this peak occurs for different values of the SBH mass $M$, spin $a / M$, pericenter $r_{p}$, orbital inclination $\iota$, and polar angle $\theta_{p}$ at pericenter.

the Killing vectors and tensor of the Kerr spacetime provide a series of first-order differential equations for the orbital motion of the tidal debris given the orbital constants. Combining these ingredients according to the prescription given at the end of Sec. III, we can calculate the fallback accretion rate $d m / d t$ as a function of time $t$.

If $d m / d t$ is greater than the Eddington accretion rate, as will be the case for $M \lesssim 10^{7} M_{\odot}$ and $t \simeq t_{\text {peak }}$, the emission may be dominated by powerful super-Eddington outflows whose luminosity will not be proportional to $d m / d t$ [53]. At later times and for larger SBH masses, the luminosity will be dominated by an accretion disk about the $\mathrm{SBH}$, whose emission may be partially reprocessed by unbound tidal debris. The bolometric luminosity of this accretion disk should be proportional to $d m / d t$, although the finite extent of the disk implies that the emission spans a fairly narrow range of temperatures 45. Monochromatic light curves may therefore not be proportional to $d m / d t$; optical emission will lie on the Rayleigh-Jeans tail of the distribution and thus scale as $(d m / d t)^{1 / 4}$ [45, 53]. Although the connection between observed monochromatic light curves and the fallback accretion rate is highly nontrivial, this accretion rate con- 
stitutes the primary input for more sophisticated calculations of TDE emission.

Our preliminary survey of relativistic tidal disruption suggests that the spin magnitude $a / M$, inclination $\iota$, and the polar angle at pericenter $\theta_{p}$ can all affect the fallback accretion rate provided $r_{p}$ is sufficiently small. The newly discovered optical transient PS1-10jh 13 may correspond to a TDE with $r_{p}=12 M$ [54] where relativistic effects can be significant as seen in Fig. 13 . The light curve associated with this event shows systematic differences with the LKP09 model during the early rise that may be sensitive to relativistic corrections; a detailed comparison between our model and this light curve is an important subject of future work.

We find that as $r_{p}$ approaches the radius of the marginally bound circular orbit (below which the star will be directly captured by the $\mathrm{SBH}$ ), relativistic corrections can increase the peak accretion rate $d m_{\text {peak }} / d t$ and reduce the time $t_{\text {peak }}$ at which this peak accretion occurs by a factor of two. For a fixed value of $r_{p}$, relativistic effects are largest for large $\mathrm{SBH}$ spins that are anti-aligned with the star's initial orbital angular momentum. However, as explored in detail in Sec. IV B. there is a great deal of degeneracy between $a / M, \iota$, and $\theta_{p}$. This degeneracy is not terribly surprising given the crudeness of our model, as each of these parameters can only increase or decrease the distribution of fallback times. Multi-frequency observations may be able to partially break this degeneracy. Comparison of Figs. 11 and 14 reveals a degeneracy between the fallback accretion rates for TDEs of stars initially on inclined orbits and those on equatorial orbits of SBHs with lower spins. Although the fallback accretion rates may be the same, the disks that subsequently form about the more highly spinning SBHs should have smaller radii and thus higher temperatures. Emission from these disks should therefore be harder than that from the disks of SBHs with lower spins. A more quantitative study of the degeneracy between relativistic parameters is another subject of potential future work.

Our model of tidal disruption, like that of LKP09, is an example of what Guillochon and Ramirez-Ruiz [55] describe as a "freezing" model in that the distribution of the orbital constants of the tidal debris is "frozen in" at pericenter. This failure to account for the redistribution of orbital constants during the small but nonzero time over which tidal disruption occurs can lead to several discrepancies in the fallback accretion rate as discussed extensively in Guillochon and Ramirez-Ruiz [55]. For fully-disruptive encounters like those considered in this paper, hydrodynamical simulations performed by Guillochon and Ramirez-Ruiz suggest that $d m / d t$ is insensitive to $r_{p}$ when it is much below the tidal radius $r_{\text {tid }}$, in contrast to the predictions of freezing models. The star's self-gravity is insufficient to hold it together at pericenter for $r_{p} \ll r_{\text {tid }}$, so it is inappropriate to apply freezing models to the unperturbed star at $r_{p}$ in this regime. Future work could explore applying relativistic freezing models to the star at $r_{\text {tid }}$ instead of $r_{p}$ as suggested by Guillochon and Ramirez-Ruiz; we would expect relativistic corrections to $d m / d t$ to be much smaller in this case.

Another problem of freezing models is that they fail to account for pressure gradients that develop when the star is tidally compressed [48, 56. These pressure gradients can redistribute material to more tightly bound orbits, leading to an increased feeding rate at early times. Our study suggests that this increase in the early rise could be misinterpreted as a relativistic effect if $r_{p}$ is small. We hope to go beyond the limitations of freezing models in future work on relativistic tidal disruption. One subject deserving special scrutiny is the conditions under which the tidal debris viscously evolves into an accretion disk about the SBH. Lense-Thirring precession 35 should delay this process for stars on inclined orbits, helping to further break some of the degeneracy between spin magnitude $a / M$ and inclination $\iota$.

Despite this need for additional work, the future prospects for using TDEs to measure SBH spins seem highly promising. The number of observed TDE candidates has increased dramatically in the past few years, and future transient surveys should provide hundreds if not thousands of additional candidates [14. A portion of these candidates, like PS1-10jh [13], should have sufficiently small $r_{p}$ such that relativistic effects will be significant. We look forward to challenging our model and its future refinements with this rich observational bounty.

Acknowledgements. We would like to thank Glennys Farrar, Suvi Gezari, James Guillochon, Giuseppe Lodato, Gabe Perez-Giz, Sterl Phinney, Enrico RamirezRuiz, Roman Shcherbakov, Nick Stone, and Linda Strubbe for useful conversations.

\section{Appendix A: Kerr metric}

In this appendix, we provide explicit formulae for quantities relevant to describing tidal disruption in the Kerr spacetime [57. In Boyer-Lindquist coordinates [58] and units where $G=c=1$, the Kerr metric takes the form

$$
\begin{aligned}
d s^{2}= & g_{\alpha \beta} d x^{\alpha} d x^{\beta} \\
= & -\left(1-\frac{2 M r}{\Sigma}\right) d t^{2}-\frac{4 M a r \sin ^{2} \theta}{\Sigma} d t d \phi \\
& +\frac{\Sigma}{\Delta} d r^{2}+\Sigma d \theta^{2}+\frac{A}{\Sigma} \sin ^{2} \theta d \phi^{2}
\end{aligned}
$$

where $M$ is the mass of the black hole, $a / M$ is its dimensionless spin, and

$$
\begin{aligned}
\Sigma & \equiv r^{2}+a^{2} \cos ^{2} \theta \\
\Delta & \equiv r^{2}+a^{2}-2 M r \\
A & \equiv\left(r^{2}+a^{2}\right)^{2}-\Delta a^{2} \sin ^{2} \theta .
\end{aligned}
$$


The inverse of this metric is given by

$$
g^{\alpha \beta}=\left(\begin{array}{cccc}
-\frac{A}{\Sigma \Delta} & 0 & 0 & -\frac{2 M a r}{\Sigma \Delta} \\
0 & \frac{\Delta}{\Sigma} & 0 & 0 \\
0 & 0 & \frac{1}{\Sigma} & 0 \\
-\frac{2 M a r}{\Sigma \Delta} & 0 & 0 & \frac{\Delta-a^{2} \sin ^{2} \theta}{\Sigma \Delta \sin ^{2} \theta}
\end{array}\right)
$$

Massive particles move on timelike geodesics of the Kerr metric with 4-velocity

$$
u^{\alpha}=\left(\begin{array}{llll}
\dot{t} \dot{r} & \dot{\theta} & \dot{\phi}
\end{array}\right)
$$

where overdots denote derivatives with respect to proper time $\tau$. The Kerr metric possesses timelike and azimuthal Killing vector fields, which we will denote by $(\partial / \partial t)^{\alpha}$ and $(\partial / \partial \phi)^{\alpha}$ following the notation of Wald [52]. This implies the existence of a conserved specific energy and angular momentum

$$
\begin{aligned}
E & \equiv-g_{\alpha \beta} u^{\alpha}\left(\frac{\partial}{\partial t}\right)^{\beta}, \\
L_{z} & \equiv g_{\alpha \beta} u^{\alpha}\left(\frac{\partial}{\partial \phi}\right)^{\beta} .
\end{aligned}
$$

The Kerr metric also possesses a Killing tensor [52, 59]

$$
K_{\alpha \beta}=\Sigma\left(l_{\alpha} n_{\beta}+n_{\alpha} l_{\beta}\right)+r^{2} g_{\alpha \beta},
$$

where

$$
\begin{aligned}
l^{\alpha} & \equiv \frac{r^{2}+a^{2}}{\Delta}\left(\frac{\partial}{\partial t}\right)^{\alpha}+\frac{a}{\Delta}\left(\frac{\partial}{\partial \phi}\right)^{\alpha}+\left(\frac{\partial}{\partial r}\right)^{\alpha} \\
n^{\alpha} & \equiv \frac{r^{2}+a^{2}}{2 \Sigma}\left(\frac{\partial}{\partial t}\right)^{\alpha}+\frac{a}{2 \Sigma}\left(\frac{\partial}{\partial \phi}\right)^{\alpha}-\frac{\Delta}{2 \Sigma}\left(\frac{\partial}{\partial r}\right)^{\alpha}(\mathrm{A}
\end{aligned}
$$

are the repeated principal null vectors found in a Petrov classification of the Weyl tensor [52, 60, 61]. This implies the existence of a third constant of motion

$$
\begin{aligned}
K & \equiv K_{\alpha \beta} u^{\alpha} u^{\beta} \\
& \equiv Q+\left(L_{z}-a E\right)^{2} .
\end{aligned}
$$

The Carter constant $Q$ is defined in this way so that in the Newtonian limit $(r \rightarrow \infty, E \rightarrow 1), Q \rightarrow L_{x}^{2}+L_{y}^{2}$, where $L_{x}$ and $L_{y}$ are the $x$ and $y$ components of the orbital angular momentum. We can also define the inclination

$$
\cos \iota \equiv \frac{L_{z}}{\sqrt{Q+L_{z}^{2}}}
$$

which in the Newtonian limit converges to the angle between the SBH spin and angular momentum $\mathbf{L}_{N}$.

Eqs. A5 and (A8), along with the normalization of the 4 -velocity $g_{\alpha \beta} u^{\alpha} u^{\beta}=-1$, can be rearranged to provide equations of motion on Kerr geodesics 62

$$
\begin{aligned}
\Sigma \dot{t}= & \frac{A E-2 M a r L_{z}}{\Delta} \\
(\Sigma \dot{r})^{2}= & {\left[E\left(r^{2}+a^{2}\right)-a L_{z}\right]^{2} } \\
& -\Delta\left[r^{2}+\left(L_{z}-a E\right)^{2}+Q\right] \\
(\Sigma \dot{\theta})^{2}= & Q-L_{z}^{2} \cot ^{2} \theta-a^{2}\left(1-E^{2}\right) \cos ^{2} \theta \\
\Sigma \dot{\phi}= & L_{z} \csc ^{2} \theta+\frac{2 M a r E-a^{2} L_{z}}{\Delta} .
\end{aligned}
$$

Evaluating the covariant derivatives of tensors in Boyer-Lindquist coordinates requires the use of Christoffel symbols $\Gamma_{\beta \gamma}^{\alpha}[52]$

$$
\begin{aligned}
\nabla_{a} T^{b_{1} \cdots b_{k}}{ }_{c_{1} \cdots c_{l}}= & \partial_{a} T^{b_{1} \cdots b_{k}}{ }_{c_{1} \cdots c_{l}} \\
& +\sum_{i} \Gamma_{a d}^{b_{i}} T^{b_{1} \cdots d \cdots b_{k}}{ }_{c_{1} \cdots c_{l}} \\
& -\sum_{j} \Gamma_{a c_{j}}^{d} T^{b_{1} \cdots b_{k}}{ }_{c_{1} \cdots d \cdots c_{l}} .
\end{aligned}
$$

The Christoffel symbols are symmetric under exchange of their lower two indices. In Boyer-Lindquist coordinates, the non-vanishing Christoffel symbols are

$$
\begin{aligned}
& \Gamma_{t t}^{r} \equiv \frac{M \Delta}{\Sigma^{3}}\left(2 r^{2}-\Sigma\right) \\
& \Gamma_{t t}^{\theta} \equiv-\frac{2 M a^{2} r \sin \theta \cos \theta}{\Sigma^{3}} \\
& \Gamma_{r r}^{r} \equiv \frac{r}{\Sigma}-\frac{r-M}{\Delta} \\
& \Gamma_{r r}^{\theta} \equiv \frac{a^{2} \sin \theta \cos \theta}{\Sigma \Delta} \\
& \Gamma_{\theta \theta}^{r} \equiv-\frac{r \Delta}{\Sigma} \\
& \Gamma_{\theta \theta}^{\theta} \equiv-\frac{a^{2} \sin \theta \cos \theta}{\Sigma} \\
& \Gamma_{\phi \phi}^{r} \equiv-\frac{\Delta \sin ^{2} \theta}{\Sigma}\left[r-\frac{M a^{2} \sin ^{2} \theta}{\Sigma^{2}}\left(2 r^{2}-\Sigma\right)\right] \\
& \Gamma_{\phi \phi}^{\theta} \equiv-\frac{\sin \theta \cos \theta}{\Sigma^{3}}\left[\left(r^{2}+a^{2}\right) A-\Sigma \Delta a^{2} \sin ^{2} \theta\right]( \\
& \Gamma_{t r}^{t} \equiv \frac{M\left(r^{2}+a^{2}\right)}{\Sigma^{2} \Delta}\left(2 r^{2}-\Sigma\right) \\
& \Gamma_{t r}^{\phi} \equiv \frac{M a}{\Sigma^{2} \Delta}\left(2 r^{2}-\Sigma\right) \\
& \Gamma_{t \theta}^{t} \equiv-\frac{2 M a^{2} r \sin \theta \cos \theta}{\Sigma^{2}} \\
& \Gamma_{t \theta}^{\phi} \equiv-\frac{2 M a r \cos \theta}{\Sigma^{2} \sin \theta} \\
& \Gamma_{t \phi}^{r} \equiv-\frac{M a \Delta \sin ^{2} \theta}{\Sigma^{3}}\left(2 r^{2}-\Sigma\right) \\
& \Gamma_{t \phi}^{\theta} \equiv \frac{2 M a r\left(r^{2}+a^{2}\right) \sin \theta \cos \theta}{\Sigma^{3}} \\
& \Gamma_{r \theta}^{r} \equiv-\frac{a^{2} \sin \theta \cos \theta}{\Sigma} \\
& \Gamma_{r \theta}^{\theta} \equiv \frac{r}{\Sigma} \\
& \Gamma_{r \phi}^{t} \equiv-\frac{M a \sin ^{2} \theta}{\Sigma \Delta}\left[\frac{2 r^{2}}{\Sigma}\left(r^{2}+a^{2}\right)+r^{2}-a^{2}\right] \\
& \Gamma_{r \phi}^{\phi} \equiv \frac{r}{\Sigma}-\frac{a^{2} \sin ^{2} \theta}{\Sigma \Delta}\left(r-M+\frac{2 M r^{2}}{\Sigma}\right) \\
& \Gamma_{\theta \phi}^{t} \equiv \frac{2 M a^{3} r \sin ^{3} \theta \cos \theta}{\Sigma^{2}} \\
& \Gamma_{\theta \phi}^{\phi} \equiv \frac{\cos \theta}{\sin \theta}\left(1+\frac{2 M a^{2} r \sin ^{2} \theta}{\Sigma^{2}}\right) \text {. }
\end{aligned}
$$




\section{Appendix B: Fermi normal coordinates}

Although the Boyer-Lindquist coordinates utilized in the previous appendix are an excellent choice for describing motion along geodesics of the Kerr spacetime, Fermi normal coordinates 63 provide a better description of the local neighborhood of a particle (such as the center of mass of a tidally disrupting star) moving along such a geodesic. These coordinates are defined by first choosing a reference point $P_{0}$ on a central geodesic $G$ as the origin. One then chooses an orthonormal tetrad of 4vectors $\boldsymbol{\lambda}_{A}$ in the tangent space at $P_{0}$, where $\boldsymbol{\lambda}_{0}$ is the tangent vector to $G$ and $\boldsymbol{\lambda}_{i}(i=1,2,3)$ are three spacelike vectors. The point $\left(\tau, X^{i}\right)(i=1,2,3)$ in Fermi normal coordinates is reached by traveling a proper time $\tau$ along $G$ to a point $h(\tau)$, then traveling a proper distance $s=\left[\sum_{i}\left(X^{i}\right)^{2}\right]^{1 / 2}$ along the orthogonal geodesic passing through $h(\tau)$ whose tangent vector is $X^{i} \boldsymbol{\lambda}_{i}$.

Marck 64 constructed an explicit tetrad of 4-vectors $\boldsymbol{\lambda}_{A}$ that are parallel propagated along timelike geodesics of the Kerr spacetime. He provided components of these 4 -vectors in the canonical symmetric orthornormal tetrad first introduced by Carter 65, but to avoid introducing yet a third set of coordinates we provide these vectors in Boyer-Lindquist coordinates. $\lambda_{0}^{\alpha}$ is simply the 4 -velocity of the center of mass, given explicitly by Eqs. (A4) and A10. The Boyer-Lindquist components of the spacelike 4 -vectors $\lambda_{i}^{\alpha}$ are

$$
\begin{aligned}
& \lambda_{1}^{t}=\frac{1}{K^{1 / 2}}\left[\frac{\alpha\left(r^{2}+a^{2}\right) r \dot{r}}{\Delta}+\beta a^{2} \sin \theta \cos \theta \dot{\theta}\right] \\
& \lambda_{1}^{r}=\frac{\alpha r}{\Sigma K^{1 / 2}}\left[E\left(r^{2}+a^{2}\right)-a L_{z}\right] \\
& \lambda_{1}^{\theta}=\frac{\beta a \cos \theta}{\Sigma K^{1 / 2}}\left(a E \sin \theta-\frac{L_{z}}{\sin \theta}\right) \\
& \lambda_{1}^{\phi}=\frac{a}{K^{1 / 2}}\left(\frac{\alpha r \dot{r}}{\Delta}+\frac{\beta \cos \theta \dot{\theta}}{\sin \theta}\right) \\
& \lambda_{2}^{t}=\frac{a}{K^{1 / 2}}\left[\frac{\left(r^{2}+a^{2}\right) \cos \theta \dot{r}}{\Delta}-r \sin \theta \dot{\theta}\right] \\
& \lambda_{2}^{r}=\frac{a \cos \theta}{\Sigma K^{1 / 2}}\left[E\left(r^{2}+a^{2}\right)-a L_{z}\right] \\
& \lambda_{2}^{\theta}=-\frac{r}{\Sigma K^{1 / 2}}\left(a E \sin \theta-\frac{L_{z}}{\sin \theta}\right) \\
& \lambda_{2}^{\phi}=\frac{1}{K^{1 / 2}}\left(\frac{a^{2} \cos \theta \dot{r}}{\Delta}-\frac{r \dot{\theta}}{\sin \theta}\right) \\
& \lambda_{3}^{t}=\alpha \frac{r^{2}+a^{2}}{\Sigma \Delta}\left[E\left(r^{2}+a^{2}\right)-a L_{z}\right] \\
& -\beta \frac{a}{\Sigma}\left(a E \sin ^{2} \theta-L_{z}\right) \\
& \lambda_{3}^{r}=\alpha \dot{r} \\
& \lambda_{3}^{\theta}=\beta \dot{\theta} \\
& \lambda_{3}^{\phi}=\frac{\alpha a}{\Sigma \Delta}\left[E\left(r^{2}+a^{2}\right)-a L_{z}\right]-\frac{\beta}{\Sigma}\left(a E-\frac{L_{z}}{\sin ^{2} \theta}\right)
\end{aligned}
$$

where

$$
\begin{aligned}
\alpha & \equiv\left(\frac{K-a^{2} \cos ^{2} \theta}{r^{2}+K}\right)^{1 / 2} \\
\beta & \equiv \frac{1}{\alpha}
\end{aligned}
$$

The basis 4 -vectors $\boldsymbol{\lambda}_{1}$ and $\boldsymbol{\lambda}_{3}$ given above are equal to $\tilde{\boldsymbol{\lambda}}_{1}$ and $\tilde{\boldsymbol{\lambda}}_{3}$ given in 64, and are not parallel propagated along the central geodesic $G$. However, since we only make use of Fermi normal coordinates at a single point along $G$ (the pericenter of the tidally disrupted star's orbit), we are justified in using these vectors in our tetrad. If the origin $P_{0}$ (the center of mass of a star) is located at $x_{0}^{\alpha}$ in Boyer-Lindquist coordinates, an event located at $\left(0, X^{i}\right)$ in Fermi normal coordinates will be located at

$$
x^{\alpha}=x_{0}^{\alpha}+X^{i} \lambda_{i}^{\alpha}
$$


[1] F. Hoyle, W. A. Fowler, Mon. Not. Roy. Astron. Soc. 125, 169 (1963).

[2] D. Lynden-Bell, Nature 223, 690 (1969).

[3] J. G. Hills, Nature 254, 295 (1975).

[4] P. J. Young, G. A. Shields, J. C. Wheeler Astrophys. J. 212, 367 (1977).

[5] A. Toomre, J. Toomre, Astrophys. J. 178, 623 (1972).

[6] J. E. Barnes, L. E. Hernquist, Astrophys. J. 370, L65 (1991).

[7] M. J. Rees, Nature 333, 523 (1988).

[8] N. Bade, S. Komossa, M. Dahlem, Astron. Astrophys. 309, L35 (1996).

[9] J. L. Donley, W. N. Brandt, M. C. Eracleous and T. .Boller, Astron. J. 124, 1308 (2002).

[10] P. Esquej, R. D. Saxton, M. J. Freyberg, A. M. Read, B. Altieri, M. Sanchez-Portal and G. Hasinger, Astron. Astrophys. 462, L49 (2007).

[11] S. Gezari, D. C. Martin, B. Milliard, S. Basa, J. P. Halpern, K. Forster, P. G. Friedman, P. Morrissey et al., Astrophys. J. 653, L25 (2006).

[12] S. Gezari, S. Basa, D. C. Martin, G. Bazin, K. Forster, B. Milliard, J. P. Halpern and P. G. Friedman et al., Astrophys. J. 676, 944 (2008).

[13] S. Gezari, R. Chornock, A. Rest, M. E. Huber, K. Forster, E. Berger, P. J. Challis and J. D. Neill et al., Nature 485 , 217 (2012).

[14] S. van Velzen, G. R. Farrar, S. Gezari, N. Morrell, D. Zaritsky, L. Ostman, M. Smith, J. Gelfand, A. J. Drake, Astrophys. J. 741, 73 (2011).

[15] D. N. Burrows, J. A. Kennea, G. Ghisellini, V. Mangano, B. Zhang, K. L. Page, M. Eracleous, P. Romano et al., Nature 476, 421 (2011).

[16] A. J. Levan, N. R. Tanvir, S. B. Cenko, D. A. Perley, K. Wiersema, J. S. Bloom, A. S. Fruchter, A. d. U. Postigo et al., Science 333, 199 (2011).

[17] J. S. Bloom, D. Giannios, B. D. Metzger, S. B. Cenko, D. A. Perley, N. R. Butler, N. R. Tanvir, A. J. Levan et al., Science 333, 203 (2011).

[18] S. B. Cenko, H. A. Krimm, A. Horesh, A. Rau, D. A. Frail, J. A. Kennea, A. J. Levan, S. T. Holland et al., Astrophys. J. 753, 77 (2012).

[19] J. M. Bardeen, Nature 226, 64 (1970).

[20] K. S. Thorne, Astrophys. J. 191, 507 (1974).

[21] R. Moderski and M. Sikora, Astron. Astrophys. Suppl. Ser. 120, 591 (1996).

[22] A. R. King, J. E. Pringle and J. A. Hofmann, Mon. Not. Roy. Astron. Soc. 385, 1621 (2008).

[23] P. F. Hopkins, L. Hernquist, C. C. Hayward and D. Narayanan, arXiv:1111.1236 [astro-ph.CO].

[24] M. Volonteri, P. Madau, E. Quataert and M. J. Rees, Astrophys. J. 620, 69 (2005)

[25] E. Berti and M. Volonteri, Astrophys. J. 684, 822 (2008).

[26] E. Barausse, Mon. Not. Roy. Astron. Soc. 423, 2533 (2012).

[27] P. W. Guilbert and M. J. Rees, Mon. Not. Roy. Astron. Soc. 233, 475 (1988).

[28] A. P. Lightman and T. R. White, Astrophys. J. 335, 57 (1988).

[29] A. C. Fabian, M. J. Rees, L. Stella and N. E. White, Mon. Not. Roy. Astron. Soc. 238, 729 (1989).

[30] A. Laor, Astrophys. J. 376, 90 (1991).
[31] L. W. Brenneman and C. S. Reynolds, Astrophys. J. 652, 1028 (2006).

[32] L. W. Brenneman, C. S. Reynolds, M. A. Nowak, R. C. Reis, M. Trippe, A. C. Fabian, K. Iwasawa and J. C. Lee et al., Astrophys. J. 736, 103 (2011).

[33] R. D. Blandford and R. L. Znajek, Mon. Not. Roy. Astron. Soc. 179, 433 (1977).

[34] W. -H. Lei and B. Zhang, Astrophys. J. 740, L27 (2011).

[35] J. Lense and H. Thirring, Phys. Z. 19, 156 (1918).

[36] N. Stone and A. Loeb, Phys. Rev. Lett. 108, 061302 (2012).

[37] W. H. Lei, B. Zhang, H. Gao, arXiv:1202.4231 [astroph.HE]].

[38] A. M. Beloborodov, A. F. Illarionov, P. B. Ivanov, A. G. Polnarev Mon. Not. Roy. Astron. Soc. 259, 209 (1992).

[39] P. B. Ivanov, M. A. Chernyakova, Astron. Astrophys. 448, 843 (2006).

[40] M. Kesden, Phys. Rev. D 85, 024037 (2012).

[41] J.-P. Luminet and J.-A. Marck, Mon. Not. Roy. Astron. Soc. 212, 57 (1985).

[42] P. Laguna, W. A. Miller, W. H. Zurek, M. B. Davies, Astrophys. J. 410, L83 (1993).

[43] P. Diener, V. P. Frolov, A. M. Khokhlov, I. D. Novikov, C. J. Pethick Astrophys. J. 479, 164 (1997).

[44] P. B. Ivanov, M. A. Chernyakova, I. D. Novikov, Mon. Not. Roy. Astron. Soc. 338, 147 (2003).

[45] G. Lodato and E. Rossi, Mon. Not. Roy. Astron. Soc. 410, 359 (2011).

[46] E. S. Phinney, in The Center of the Galaxy: Proceedings of the 136th Symposium of the International Astronomical Union, edited by M. Morris (Kluwer Academic Publishers, Dordrecht, 1989), p. 543.

[47] S. Komossa and N. Bade, Astron. Astrophys. 343, 775 (1999).

[48] G. Lodato, A. R. King and J. E. Pringle, Mon. Not. Roy. Astron. Soc. 392, 332 (2009).

[49] R. Haas, R. V. Shcherbakov, T. Bode and P. Laguna, Astrophys. J. 749, 117 (2012).

[50] C. R. Evans and C. S. Kochanek, Astrophys. J. 346, L13 (1989).

[51] S. L. Shapiro and S. A. Teukolsky, Black Holes, White Dwarfs, and Neutron Stars: The Physics of Compact Objects (John Wiley \& Sons, Inc., New York, 1983).

[52] R. M. Wald, General Relativity (University of Chicago Press, Chicago, 1984).

[53] L. E. Strubbe and E. Quataert, Mon. Not. Roy. Astron. Soc. 400, 2070 (2009).

[54] G. Lodato, Nature 485, 183 (2012).

[55] J. Guillochon and E. Ramirez-Ruiz, arXiv:1206.2350 [astro-ph.HE].

[56] J. Guillochon, E. Ramirez-Ruiz, S. Rosswog, and D. Kasen, Astrophys. J. 705, 844 (2009).

[57] R. P. Kerr, Phys. Rev. Lett. 11, 237 (1963).

[58] R. H. Boyer and R. W. Lindquist, J. Math. Phys. 8, 265 (1967).

[59] M. Walker and R. Penrose, Commun. Math. Phys. 18, 265 (1970).

[60] A. Z. Petrov, Scientific Proceedings of Kazan State University 114 (8), 55 (1954).

[61] A. Z. Petrov, Einstein spaces (Pergamon Press, Oxford, 
1969).

[62] B. Carter, Phys. Rev. 174, 1559 (1968).

[63] F. K. Manasse and C. W. Misner, J. Math. Phys. 4, 735 (1963).
[64] J.-A. Marck, Proc. R. Soc. Lond. A 385, 431 (1983).

[65] B. Carter, Commun. Math. Phys. 10, 280 (1968). 